\title{
Choice and prohibition in non-monotonic contexts
}

\author{
Nicole Gotzner ${ }^{1}$. Jacopo Romoli ${ }^{2}\left([) \cdot\right.$ Paolo Santorio $^{3}$ \\ Published online: 4 March 2020 \\ (c) The Author(s) 2020
}

\begin{abstract}
Disjunctions in the scope of possibility modals give rise to a conjunctive inference, generally referred to as 'free choice.' For example, Emma can take Spanish or Calculus suggests that Emma can take Spanish and can take Calculus. This inference is not valid on standard semantics for modals in combination with a Boolean semantics for disjunction. Hence free choice has sparked a whole industry of theories in philosophy of language and semantics. This paper investigates free choice in sentences involving a non-monotonic modified numeral, under which we embed a possibility modal scoping over disjunction. One example is Exactly one student can(not) take Spanish or Calculus. As we point out, the presence (or absence) of certain readings of these sentences is a key test for a prominent approach, which analyzes free choice as a kind of scalar implicature. We report on two experiments investigating the readings of such sentences, using an inferential task. Our results are challenging for the implicature approach. We sketch two possible solutions within this approach, either adopting a different recent implicature algorithm, or exploring a different meaning for modified numerals with exactly. Both of them suffer from a variety of problems. We then discuss a third solution, which exploits a recent account of free choice based on homogeneity. This approach can account for our results, in combination with plausible assumptions about homogeneity projection, though it too has open issues with related cases. Regardless of which solution is chosen, non-monotonic contexts turn out to be an important test case for theories of free choice, implicature, and modified numerals.
\end{abstract}

Keywords Free choice $\cdot$ Modified numerals $\cdot$ Implicatures $\cdot$ Homogeneity

\section{Introduction}

Disjunctions in the scope of possibility modals give rise to a conjunctive inference, generally referred to as 'free choice' (Kamp 1974 and much subsequent work). For

Nicole Gotzner

gotzner@ leibniz-zas.de

Extended author information available on the last page of the article 
example, (1a) suggests that Emma can take Spanish and can take Calculus (and hence that she can 'choose' between the two; (1b)).

a. Emma can take Spanish or Calculus.

b. $\rightsquigarrow$ Emma can choose between the two

The inference in (1b) is problematic, since it is not validated by a classical semantics for modals in combination with a Boolean analysis of disjunction. To complicate things further, free choice tends to disappear under negation: (2) doesn't merely suggests that Emma can't choose, but rather that she can take neither Spanish nor Calculus. This second effect is sometimes (see e.g. Goldstein 2018) referred to as 'Dual prohibition.'

a. Emma cannot take Spanish or Calculus.

b. $\rightsquigarrow$ Emma can take neither of the two

The free choice-dual prohibition pattern has sparked a whole industry of theories in philosophy of language and semantics since the seventies. ${ }^{1}$ A theory of this pattern must predict how free choice arises in positive contexts and how it gives place to dual prohibition in negative ones.

In this paper, we investigate this pattern in sentences involving a non-monotonic modified numeral and a possibility modal which scopes over disjunction. We focus on sentences like (3) and (4).

(3) Exactly one student can take Spanish or Calculus.

(4) Exactly one student cannot take Spanish or Calculus.

In particular, we investigate whether (3) has a reading suggesting that one student can choose between Spanish and Calculus and all other students can take neither of the two. In other words, on this reading one student has free choice and each of the others is subject to dual prohibition. We call this reading 'ALL-OTHERS-DUAL-PROHIBITION'. Analogously, we ask whether (4) has a corresponding reading on which one student is subject to dual prohibition and each of the others has free choice; we call this reading 'ALL-OTHERS-FREE-CHOICE.'

As we discuss below, the presence (or absence) of these readings is a key test for a prominent approach to free choice, which analyzes free choice as emerging from the application of a scalar implicature-type reasoning. ${ }^{2}$ This approach predicts ALLOTHERS-DUAL-PROHIBITION as a possible reading for (3), but it does not predict the corresponding ALL-OTHERS-FREE-CHOICE to be a possible reading of (4). Hence, if the scalar approach is correct, we should find evidence for the former but not for the latter.

In this paper, we report on two experiments investigating this prediction, using an inferential task. We find empirical evidence for both readings, which is a challenging result for the standard scalar implicature approach to free choice.

\footnotetext{
${ }^{1}$ See Meyer (2018) and references therein.

2 See Kratzer and Shimoyama (2002), Alonso Ovalle (2005), Fox (2007), Bar-Lev and Fox (2017), Bar-Lev (2018), Klinedinst (2007), Franke (2011), Santorio and Romoli (2017), Chemla (2010), among others.
} 
After pointing out the problem, we turn to investigating solutions. We first sketch two options that retain the implicature approach. The first adopts a different algorithm for implicature computation, which has recently been proposed by Bar-Lev and Fox (2017) and Bar-Lev (2018). The second employs a different meaning for modified numerals with exactly, based on suggestions in the literature (Landman 1998; Spector 2014). Both of them suffer from a variety of problems at this stage. We then turn to a third solution, based on a homogeneity account of free choice (Goldstein 2018). This solution can account for our results, given certain assumptions about the projection of homogeneity in complex sentences (Križ 2015; Križ and Chemla 2015; among others), though it too leaves a variety of issues open with related cases.

Regardless of which solution is chosen, our discussion shows that non-monotonic contexts are a fertile area of inquiry for making progress on free choice, scalar implicatures, and modified numerals.

The rest of the paper is organised as follows: In Sect. 2, we sketch the free choice and dual prohibition pattern in more detail, both for the basic and the non-monotonic cases. We also briefly discuss the standard implicature approach and its main predictions in relation to such cases. In Sects. 3 and 4, we report on the two experiments investigating these predictions. In Sects. 5 and 6, we discuss why our results are challenging for the predictions of the standard implicature approach and outline the options for a solution.

\section{Background}

\subsection{Free choice, dual prohibition, and non-monotonic contexts}

A positive sentence with a simple non-quantified subject like (1a), repeated here as (5a), gives rise to the inference in (5b). Conversely, its negation in (2a), repeated here in (6a), doesn't merely convey the negation of free choice, but rather the stronger dual prohibition inference in (6b).

a. Emma can take Spanish or Calculus.

b. $\rightsquigarrow$ Emma can choose between the two

a. Emma cannot take Spanish or Calculus.

b. $\rightsquigarrow$ Emma can take neither of the two

Schematically: a sentence of the form of (7a) triggers the inference in (7b), while its negation in (8a) triggers the inference in (8c), rather than the weaker one in (8b).

$$
\begin{aligned}
& \text { a. } \diamond(\mathrm{S} e \vee \mathrm{C} e) \\
& \text { b. } \nabla \mathrm{S} e \wedge \nabla \mathrm{C} e \\
& \text { (8) a. } \neg \diamond(\mathrm{S} e \vee \mathrm{C} e) \\
& \text { b. } \neg \diamond \mathrm{Se} \vee \neg \vee \mathrm{C} e \\
& \text { c. } \neg \diamond \mathrm{S} e \wedge \neg \vee \mathrm{C} e
\end{aligned}
$$

FREE CHOICE

NEGATED FREE CHOICE DUAL PROHIBITION

Consider now the non-monotonic cases in (3) and (4), repeated below in (9) and (10). We can ask how the basic pattern applies to them. In particular, we want to ask whether 
(9a) has a reading suggesting that there is one student who has free choice between Spanish and Calculus, while each of the others is subject to dual prohibition (as in (9b)). Similarly, for (10a), we want to ask whether it has a reading conveying that one student is subject to dual prohibition and each of the others has free choice (as in (10b)).

(9) a. Exactly one student can take Spanish or Calculus.

b. ? $\rightsquigarrow$ One student can choose between the two and each of the others can take neither of them

(10) a. Exactly one student cannot take Spanish or Calculus.

b. ? $\rightsquigarrow$ One student can take neither Calculus nor Spanish and each of the others can choose between them

More schematically, we can write (9a) as in (11a) and the reading we are interested in as in $(11 \mathrm{~b}) .^{3}$

$$
\begin{aligned}
& \text { a. } \exists x^{|x|=1}[\diamond(S x \vee C x)] \\
& \text { b. } \exists x^{|x|=1}\left[(\diamond S x \wedge \diamond C x) \wedge \forall y^{y \neq x}[(\neg \diamond S y \wedge \neg \diamond C y)]\right]
\end{aligned}
$$

To sharpen intuitions, consider a model with three individuals: Ann, Bill, and Carl. This reading of $(9 a)$ would convey that we are in a situation like the one described in (12).

\begin{tabular}{|c|c|c|}
\hline Ann & $\diamond \mathrm{S} a \wedge \triangleright \mathrm{C} a$ & FREE CHOICE \\
\hline Bill & $\neg \diamond \mathrm{S} b \wedge \neg \diamond \mathrm{C} b$ & DUAL PROHIBITION \\
\hline Carl & $\neg \diamond \mathrm{S} c \wedge \neg \diamond \mathrm{C} c$ & DUAL PROHIBITION \\
\hline
\end{tabular}

Similarly, we can represent schematically (10a) as in (13a) and its potential reading as in (13b).

$$
\begin{aligned}
& \text { a. } \exists x^{|x|=1}[\neg \diamond(\mathrm{S} x \vee \mathrm{C} x)] \\
& \text { b. } \exists x^{|x|=1}\left[(\neg \diamond \mathrm{S} x \wedge \neg \vee \mathrm{C} x) \wedge \forall y^{y \neq x}[(\diamond \mathrm{S} y \wedge \diamond \mathrm{C} y)]\right]
\end{aligned}
$$

If (10a) has this reading, it will convey that we are in a situation like the one in (14).

\begin{tabular}{|c|c|c|}
\hline Ann & $\neg \diamond \mathrm{S} a \wedge \neg \diamond \mathrm{C} a$ & DUAL PROHIBITION \\
\hline Bill & $\diamond \mathrm{S} b \wedge \diamond \mathrm{C} b$ & FREE CHOICE \\
\hline Carl & $\diamond \mathrm{S} c \wedge \diamond \mathrm{C} c$ & FREE CHOICE \\
\hline
\end{tabular}

The case in (9a) and its potential reading in (11b) has been discussed in the literature (Bassi and Bar-Lev 2016, among others), while (10a) is, to our knowledge, a novel test case. In addition, neither of the readings has been investigated experimentally. In the next subsection, we sketch the standard implicature approach and outline the predictions it makes for the non-monotonic pair above.

\footnotetext{
${ }^{3} \operatorname{Read} \exists x^{|x|=1}$ as $\exists x\left[|x|=1\right.$ and $\forall y^{y \neq x}$ as $\forall y[y \neq x$.
} 


\subsection{The scalar implicature approach and its predictions}

\subsubsection{The basic pattern}

A prominent approach in the literature analyzes free choice as a kind of scalar implicature. The implicature approach assumes that the basic meaning of a sentence like (1a) is the one predicted by classical semantics for modals and disjunction. On this meaning, Emma can take at least one of Spanish and Calculus, as represented in (15).

$$
\diamond(\mathrm{Se} \vee \mathrm{C} e)=\diamond \mathrm{S} e \vee \diamond \mathrm{C} e
$$

LITERAL MEANING

Of course, this does not predict free choice. But it immediately predicts dual prohibition: the negation of (15) is equivalent to the conjunction of the negations of the two relevant possibility claims.

$$
\neg \diamond(\mathrm{S} e \vee \mathrm{C} e)=\neg \diamond \mathrm{S} e \wedge \neg \vee \mathrm{C} e
$$

DUAL PROHIBITION

There are several versions of the implicature approach (see fn. 3 above). But all of them make use of two crucial assumptions. First, they adopt the assumption that the basic meaning in (15) can be strengthened via an appropriate algorithm for computing scalar implicatures, in a way that predicts free choice. Let us for now use 'IMP' to represent an implicature-computing algorithm that takes sentences to their strengthened meanings. The implicature theorist assumes, schematically:

$$
\operatorname{IMP}(\diamond(\mathrm{S} e \vee \mathrm{C} e))=\diamond \mathrm{S} e \wedge \diamond \mathrm{C} e
$$

FREE CHOICE

Second, the implicature approach assumes that the implicature-computing algorithm is not always available. In particular, its use is disallowed, or at least strongly dispreferred, in downward entailing contexts like the one generated by negation. This blocks the configuration in (18). ${ }^{4}$

$$
\# / ? ? \neg \operatorname{IMP}(\diamond(\mathrm{S} e \vee \mathrm{C} e))
$$

NEGATED FREE CHOICE

With these ingredients in place, the implicature approach can account for the free choice-dual prohibition pattern in the basic case.

The main arguments for this approach come from the distributional analogies between free choice and scalar implicature. In particular, both free choice and implicature display sensitivity to polarity, i.e. they are sensitive to whether an environment is monotonic or not. This is a signature feature of implicatures, which have a strong tendency to disappear in non-monotonic contexts. Another argument for the implicature approach comes from the well-known observation that free choice inferences are cancellable. ${ }^{5}$ That is, while (1a) strongly suggest a free choice inference, we can, in the right context, force a reading without this inference. For example, we can add a continuation incompatible with this inference, as in (19).

\footnotetext{
${ }^{4}$ For discussion of the constraints on the distribution of EXH see Chierchia et al. (2012), Fox and Spector (2018), among others.

5 See Simons (2005), Fox (2007), among others.
} 
(19) Emma can take Spanish or Calculus. I don't remember which.

The implicature approach nicely captures these and other related data points. ${ }^{6}$

In the following, we focus on a debate within the implicature approach. The key issue is what mechanism for implicature computation will generate free choice. The standard implicature approach derives implicatures by 'excluding' alternatives, i.e. by conjoining negations of alternatives to the content of the sentence asserted. We use Fox (2007) for illustration, but the same conclusions apply to all exclusion accounts we are aware of.

Fox (2007) starts from a grammatical approach to implicatures. ${ }^{7}$ On this approach, the implicature generating algorithm works via a covert exhaustifying operator, standardly referred to as 'EXH', which is present in the syntax. EXH takes as arguments a sentence and a set of alternatives and returns the conjunction of the sentence with the negation of a subset of the alternatives - i.e., the alternatives that are 'innocently excludable' (more on this notion below). Informally, EXH looks at all the maximal consistent subsets of alternatives to a sentence, and negates all alternatives that are in all those subsets. Roughly, the effect is to strengthen the sentence as much as possible, while avoiding contradictions and arbitrary choices between alternatives. The meaning of EXH is given in (20), while the definition of innocent exclusion is in (21), where ' $C$ ' stands for a set of salient alternatives to a sentence.

$$
\begin{aligned}
& \llbracket \operatorname{EXH} \rrbracket(A)(p)(w)=p_{w} \wedge \forall q \in \operatorname{IE}(p, A)\left[\neg q_{w}\right] \\
& \mathbb{I E}(p, C):=\bigcap\left\{\begin{array}{l|l}
C^{\prime} & \begin{array}{l}
C^{\prime} \subseteq C \text { and } C^{\prime} \text { is a maximal subset of } C \\
\text { such that }\{\neg q: q \in C)\} \cup\{p\} \text { is consistent }
\end{array}
\end{array}\right\}
\end{aligned}
$$

Let us illustrate how this derives the exclusive reading of disjunction as a scalar implicature in a simple sentence like (22).

\section{Emma took Spanish or Calculus.}

$\rightsquigarrow$ Emma didn't take both Spanish and Calculus

(22) is parsed as involving a covert exhaustivity operator, as in (23). We assume that the alternatives of (22) are those in (24). ${ }^{8}$

$$
\text { EXH[Emma took Spanish or Calculus] }
$$

$\left\{\begin{array}{lr}\text { Emma took Spanish or Calculus } & (\mathrm{S} e \vee \mathrm{C} e) \\ \text { Emma took Spanish } & \mathrm{Se} \\ \text { Emma took Calculus } & \mathrm{C} e \\ \text { Emma took Spanish and Calculus }(\mathrm{Se} \wedge \mathrm{Ce} e\end{array}\right\}$

\footnotetext{
6 There are also a variety of challenges for this approach stemming from certain differences between implicatures and free choice in their processing and acquisition profiles (Chemla and Bott 2014; Tieu et al. 2016). Other challenges stem from the interaction between free choice and presuppositions (Romoli and Santorio 2019; Marty and Romoli 2019) and the status of positive versus negative sentences in certain contexts (Tieu et al. 2018).

7 See Chierchia (2004, 2013), Chierchia et al. (2012), Magri (2009), Meyer (2013), among others.

8 How the alternatives for exhaustification are determined is an important issue for all implicature accounts. In fact, this is a controversial point; we will return to it later in Sect. 5. For relevant discussion see Breheny et al. (2018) and references therein.
} 
Given the alternatives in (24), only the conjunctive alternative ( $\mathrm{S} e \wedge \mathrm{C} e$ ) is excludable. This is because there are only two maximal consistent subsets of excludable alternatives, $\{\mathrm{Se}, \mathrm{S} e \wedge \mathrm{C} e\}$ and $\{\mathrm{C} e, \mathrm{~S} e \wedge \mathrm{C} e\}$, and only the conjunctive alternative appears in both. This yields the intuitively correct prediction, i.e. the implicature in (22).

This algorithm, by itself, is insufficient to derive free choice, but it can be enriched in a number of ways. Fox (2007) derives free choice by adding EXH recursively. ${ }^{9}$ For illustration, consider again (1a). Crucially, Fox assumes that (1a) is parsed as involving two occurrences of EXH. ${ }^{10}$ On this parsing, the outermost EXH operates on alternatives that have already been exhaustified by the innermost EXH. In particular, the alternatives include the exhaustified disjuncts, which are innocently excludable. ${ }^{11}$ The negation of these two alternatives, together with the content of the assertion, gives rise to the free choice effect (see the schematic computation in (27)).

EXH[EXH[Emma can take Spanish or Calculus]]

\begin{tabular}{|c|c|}
\hline $\mathrm{EXH}[$ Emma can take $\mathrm{S}$ or $\mathrm{C}]$ & $\diamond(\mathrm{S} e \vee \mathrm{C} e) \wedge \neg \diamond(\mathrm{S} e \wedge \mathrm{C} e)$ \\
\hline EXH[Emma can take $\mathrm{S}]$ & \\
\hline $\mathrm{EXH}[$ Emma can take $\mathrm{C}$ & \\
\hline $\mathrm{EXH}[$ Emma can take $\mathrm{S}$ and $\mathrm{C}]$ & $\diamond(\mathrm{S} e \wedge \mathrm{C} e)$ \\
\hline
\end{tabular}
【EXH[EXH[Emma can take Spanish or Calculus]]》 = $\diamond(\mathrm{S} e \vee \mathrm{C} e) \wedge \neg[\nabla \mathrm{S} e \wedge \neg \vee \mathrm{C} e] \wedge \neg[\diamond \mathrm{C} e \wedge \neg \diamond \mathrm{S} e]=$ $\diamond(\mathrm{S} e \vee \mathrm{C} e) \wedge \diamond \mathrm{S} e \leftrightarrow \nabla \mathrm{C} e=$ $\diamond(\mathrm{S} e \vee \mathrm{C} e) \wedge \diamond \mathrm{S} e \wedge \diamond \mathrm{C} e$

In the next subsection, we turn to the predictions of this approach for the non-monotonic cases we introduced in Sect. 1.

\subsubsection{The non-monotonic cases}

Consider again (9a) and (10a) and their potential readings. Let us explore the predictions of the implicature account, starting with the ALL-OTHERS-DUAL-PROHIBITION reading. Suppose that we parse (9a) as in (28). It is routine to check that, if we parse the sentence as involving only one exhaustivity operator, only the conjunctive alternative is innocently excludable. But, as it happens for our basic case of free choice (22), we can derive free choice if we exhaustify recursively. ${ }^{12}$ Suppose we parse the sentence as:

\section{EXH[EXH[Exactly one student can take Spanish or Calculus]]}

\footnotetext{
9 For alternative accounts, see fn. 3 above.

10 On the parsing that involves only one occurrence of EXH, the prediction is that we simply exclude the conjunctive alternative, exactly as happens for (22).

11 There is only one maximal excludable set of alternatives, $\{\diamond S \wedge \neg \diamond C, \diamond C \wedge \neg \diamond S, \diamond(S \wedge C)\}$.

12 See Spector (2007) for a similar case with the inference of plurals.
} 
Using the schematic representation for the prejacent, $\exists x^{|x|=1}[\diamond(S x \vee C x)]$, we can represent the alternatives as in (29). ${ }^{13}$

$$
\left\{\begin{array}{lr}
\text { EXH[Exactly one can take S or C] } & \exists x^{|x|=1}[\diamond(\mathrm{S} x \vee \mathrm{C} x)] \\
\mathrm{EXH}[\text { Exactly one can take S }] & \exists x^{|x|=1}[\diamond \mathrm{S} x] \wedge \neg \exists x^{|x|=1}[\diamond \mathrm{C} x] \\
\text { EXH[Exactly one can take C] } & \exists x^{|x|=1}[\diamond \mathrm{C} x] \wedge \neg \exists x^{|x|=1}[\diamond \mathrm{S} x]
\end{array}\right\}
$$

As we saw already for the derivation of free choice in simple sentences, the two alternatives involving only one disjunct are innocently excludable. The conjunction of the assertion and the negation of those two alternatives yields the ALL-OTHERSDUAL-PROHIBITION reading.

$$
\begin{aligned}
& \exists x^{|x|=1}[\diamond(S x \vee C x)] \wedge \neg\left(\exists x^{|x|=1}[\diamond S x] \wedge \neg \exists x^{|x|=1}[\diamond C x]\right) \wedge \neg\left(\exists x^{|x|=1}[\diamond C x] \wedge\right. \\
& \left.\neg \exists x^{|x|=1}[\diamond S x]\right)= \\
& \exists x^{|x|=1}[\diamond(S x \vee C x)] \wedge \exists x^{|x|=1}[\diamond S x] \leftrightarrow \exists x^{|x|=1}[\diamond C x]= \\
& \exists x^{|x|=1}[\diamond(S x \vee C x)] \wedge \exists x^{|x|=1}[\diamond S x] \wedge \exists x^{|x|=1}[\diamond C x]
\end{aligned}
$$

For illustration, consider again the toy model with Ann, Bill, and Carl; we will show now that (30) entails that we are in a situation like the one represented in Table 1 . The first conjunct of (30) is the prejacent: as we saw, this by itself conveys that there is one student who can take at least one of Spanish and Calculus, and that all the others can take neither. The second and third conjuncts (looking at the first line) say that there isn't an individual who is allowed to take one class but not the other. Since there is only one individual who is allowed to take any class, this last clause ensures that this individual has free choice between Spanish and Calculus.

Crucially, this reasoning cannot be replicated to derive ALL-OTHERS-FREE-CHOICE. For brevity, we only consider the case where we exhaustify recursively. (It's easy to check that one round of exhaustification also won't work.) We assume the parsing in (31) and the alternatives in (32).

\section{EXH[ EXH[Exactly one student cannot take Spanish or Calculus] ]}

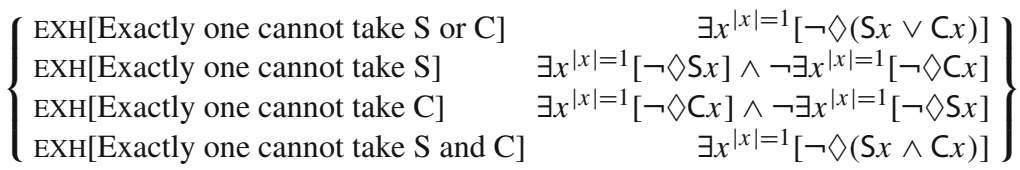

The relevant alternatives (i.e. the ones involving just the two disjuncts) are innocently excludable, as we observed for the positive case. But in this case the strengthened meaning we get is not sufficient to generate free choice. The meaning is the following:

$$
\begin{aligned}
& \exists x^{|x|=1}[\neg \diamond(S x \vee C x)] \wedge \neg\left(\exists x^{|x|=1}[\neg \diamond S x] \wedge \neg \exists x^{|x|=1}[\neg \diamond C x]\right) \wedge \neg\left(\exists x^{|x|=1}[\neg \diamond\right. \\
& \left.C x] \wedge \neg \exists x^{|x|=1}[\neg \diamond S x]\right)= \\
& \exists x^{|x|=1}[\neg \diamond(S x \vee C x)] \wedge\left(\exists x^{|x|=1}[\neg \diamond S x] \leftrightarrow \exists x^{|x|=1}[\neg \diamond C x]\right)
\end{aligned}
$$

Informally, (33) says: exactly one student is not allowed to take Spanish or Calculus, and it's not the case that: exactly one is not allowed to take Spanish and not exactly

\footnotetext{
13 We ignore here the conjunctive alternative Exactly one can take Spanish and Calculus, which leads to some irrelevant complications and choice points.
} 
Table 1 A situation which makes the predicted reading of (31) true, given a model with three individuals: Ann, Bill and Carl

\begin{tabular}{lll}
\hline Ann & $\neg \diamond \mathrm{S} a \wedge \neg \diamond \mathrm{C} a$ & DUAL PROHIBITION \\
Bill & $\diamond \mathrm{S} b \wedge \neg \diamond \mathrm{C} b$ & ONLY ONE \\
Carl & $\neg \diamond \mathrm{S} c \wedge \diamond \mathrm{C} c$ & ONLY ONE \\
\hline
\end{tabular}

one is not allowed to take Calculus, and it's not the case that: exactly one is not allowed to take Calculus and not exactly one is not allowed to take Spanish. These truth conditions hold in a scenario like the one in Table 1 in which one student is subject to dual prohibition, and each of the others can only take one of the two.

That is, (33) does not entail the ALL-OTHERS-FREE-CHOICE reading. The first conjunct of (33) is made true by the fact that Ann is subject to dual prohibition, plus the fact that no other student is. The biconditional is made true by the fact that more than one student can take Calculus and more than one student can take Spanish. (Hence both terms of the biconditional are false, which makes the biconditional as a whole true.)

In sum, the standard implicature approach makes the predictions in (34): it predicts that (9a) can have the ALL-OTHERS-DUAL-PROHIBITION reading, while (10a) cannot have the corresponding ALL-OTHERS-FREE-CHOICE reading. So we should find evidence for the former but not the latter.

Predictions of the standard implicature approach:

a. ALL-OTHERS-DUAL-PROHIBITION $\checkmark$

b. ALL-OTHERS-FREE-CHOICE $\times$

In the following, we turn to two experiments investigating these predictions. Before that, for completeness, we briefly sketch some other possible readings of sentences like (9a) and (10a), and we discuss their relationship to the ALL-OTHERS-DUALPROHIBITION and ALL-OTHERS-FREE-CHOICE readings we are focusing on.

\subsection{Other readings}

The readings examined above are of course not the only potential readings of our target examples. Let us mention a couple of other salient readings. We illustrate them using the positive case. The first is simply the LITERAL reading, which is the reading we get by assuming that the modal and disjunction have classical meanings, and that the sentence has no implicatures. We have already seen this meaning: it entails that one student can take at least one of the two classes, while each of the others has dual prohibition.

a. Exactly one student can take Spanish or Calculus.

b. $\rightsquigarrow$ One student can take at least one of the two and each of the others can take neither of them

LITERAL

Schematically, the reading of (35a) can be written as in (36b). 


$$
\begin{aligned}
& \text { a. } \exists x^{|x|=1}[\diamond(S x \vee C x)] \\
& \text { b. } \exists x^{|x|=1}\left[(\diamond S x \vee \diamond C x) \wedge \forall y^{y \neq x}[(\neg \diamond S y \wedge \neg \diamond C y)]\right]
\end{aligned}
$$

LITERAL

The second possible reading is the one we call LOCAL. This is the reading we obtain in Fox's account by embedding two occurrences of EXH in the scope of exactly one, as in (37a).
a. Exactly one student[EXH[EXH[can take Spanish or Calculus]]]
b. $\rightsquigarrow$ One student can choose between the two and each of the others cannot choose between the two
LOCAL

Schematically, the truth conditions of (37a) are as in (38): they entail that one student has free choice and all of the others do not have free choice.

$$
\begin{aligned}
& \text { a. } \exists x^{|x|=1}[\diamond(S x \vee C x)] \\
& \text { b. } \exists x^{|x|=1}\left[(\diamond S x \wedge \diamond C x) \wedge \forall y^{y \neq x}[(\neg \diamond S y \vee \neg \diamond C y)]\right]
\end{aligned}
$$

LOCAL

Now consider again the ALL-OTHERS-DUAL-PROHIBITION reading, repeated in (39b). Notice that this reading results from conjoining the strongest parts of each of the other two readings.

$$
\begin{aligned}
& \text { a. } \exists x^{|x|=1}[\diamond(\mathrm{S} x \vee \mathrm{C} x)] \\
& \text { b. } \exists x^{|x|=1}\left[(\diamond \mathrm{S} x \wedge \diamond \mathrm{C} x) \wedge \forall y^{y \neq x}[(\neg \diamond \mathrm{S} y \wedge \neg \vee C y)]\right]
\end{aligned}
$$

ALL-OTHERS-P

As a result, the relationships between these readings are as follows: the LOCAL and the LITERAL readings are logically independent, while the ALL-OTHERS-DUALPROHIBITION reading entails both of them. ${ }^{14}$

With this in the background, we can move to the experiments testing the predictions in (34).

\footnotetext{
${ }^{14}$ Finally, consider what happens if we allow disjunction to take wide-scope with respect to the nonmonotonic quantifier so that (i) would convey the suggested paraphrase below, schematised in (ii).

(i) Exactly one student can take Spanish or Calculus.

$\rightsquigarrow$ Either exactly one student can take Spanish or exactly one student can take Calculus

(ii) $\exists x^{|x|=1}\left[\diamond S x \wedge \forall y^{y \neq x}[(\neg \diamond S y]] \vee \exists x^{|x|=1}\left[\diamond C x \wedge \forall y^{y \neq x}[(\neg \diamond C y]]\right.\right.$ WIDE SCOPE DISJUNCTION
}

This is very weak and in fact true in a situation in which nobody has free choice and nobody has dual prohibition either, (e.g. it is true if there is one student who can take Spanish and each of the others cannot take Spanish). Relatedly, we could consider a reading in which the modal takes scope over the non-monotonic quantifier, as in (iii).

(iii) Exactly one student can take Spanish or Calculus.

$\rightsquigarrow$ It allowed that exactly one student take Spanish or Calculus

WIDE-SCOPE MODAL

Again, this would be an extremely weak reading which would not allow us to conclude that anybody has free choice or dual prohibition, and therefore would not lead to endorse any of the target inferences in the experiment below. Thanks to an anonymous reviewer for discussion on this case. 


\section{Experiment 1}

\subsection{Goals and predictions}

The goal of Experiment 1 was to investigate whether disjunctions in non-monotonic contexts give rise to the free choice readings discussed above. In our study, we employ an inferential task that builds on recent experimental work on implicatures (in particular Chemla and Spector 2011; Gotzner and Romoli 2017). Our experimental design is based on a variant of the inferential task introduced in Gotzner and Romoli (2017).

In particular, the experiment uses a $2 \times 4$ design comparing 2 polarity conditions (POSITIVE and NEGATIVE representing variants of the two sentences (3) and (4)) across four inference conditions (TRUE, FALSE, COMPATIBLE, and FREE-CHOICE). The factor 'polarity' pits the existence of different candidate in POSITIVE and NEGATIVE environments against each other. The conditions TRUE, FALSE, COMPATIBLE are baselines for the endorsement of patently true and false inferences as well as statements that are merely compatible with the test statements (but not an inference that follows). The TARGET FREE CHOICE condition is the critical one representing the endorsement of the respective free choice reading. Critically, we compare the target FREE CHOICE condition against baselines across POSITIVE and NEGATIVE environments. The prediction is that if the readings discussed above exist, in the POSITIVE or NEGATIVE case the TARGET condition should be endorsed to a higher extent than the corresponding FALSE and COMPATIBLE conditions (while the TRUE condition should receive highest endorsement rates overall).

As discussed, the standard implicature approach predicts the ALL-OTHERS-DUALPROHIBITION reading for the POSITIVE (3) but not the corresponding ALL-OTHERSFREE-CHOICE reading for the NEGATIVE condition (4). Accordingly, endorsements of the FREE CHOICE condition should be higher than for the COMPATIBLE and FALSE conditions only in the POSITIVE variant (i.e., there should be an interaction between polarity and inference condition).

\subsection{Methods}

\subsubsection{Participants}

We recruited 60 Participants with U.S. IP addresses via Amazon's Mechanical Turk and screened them for native language. They received 80 cents for participation in the study. All participants indicated their native language to be English (27 male, 31 female, 2 no gender specified, mean age 35.6).

\subsubsection{Materials}

Participants saw sentences like (3) and (4) across the four inference conditions, TRUE, FALSE, COMPATIBLE, and the TARGET FREE CHOICE condition. Each participant saw all experimental conditions in four different scenarios, totaling 16 experimental items. The order of presentation was pseudo-randomized for each scenario. 


\section{Context:}

We are at the beginning of the year waiting for the teacher to give instructions on what classes we can take. The school
offers a number of classes such as Algebra, Calculus, Logic, Physics, Chemistry, Literature, History, Spanish, French and English.

We know already that no student is allowed to take all classes. The teacher gives further instructions:

1. "Exactly one student can take Algebra or Literature"

suggests that

Only one student can take Algebra or Literature

$\%$ YES

$0 \%=$ definitely not, $100 \%=$ definitely yes

Fig. 1 Screenshot of one of the trials of Experiment 1

Table 2 Example stimuli for Experiment 1

\begin{tabular}{|c|c|c|}
\hline Inf & Pol & Candidate inference \\
\hline True & $\mathrm{p}$ & Only one student can take Spanish or Calculus \\
\hline $\mathrm{Fc}$ & $\mathrm{p}$ & $\begin{array}{l}\text { One student can choose between Spanish and Calculus, and all others can take } \\
\text { neither one }\end{array}$ \\
\hline Comp+ & $\mathrm{p}$ & One student can take either Spanish or Calculus, and all others can take logic \\
\hline Comp- & $\mathrm{p}$ & One student can take either Spanish or Calculus, and all others cannot take logic \\
\hline False & $\mathrm{p}$ & No student can take Spanish and no student can take Calculus \\
\hline True & $\mathrm{n}$ & Only one student cannot take Spanish or Calculus \\
\hline $\mathrm{Fc}$ & $\mathrm{n}$ & $\begin{array}{l}\text { One student can take neither Spanish nor Calculus, and all others can choose } \\
\text { between the two }\end{array}$ \\
\hline Comp+ & $\mathrm{n}$ & One student can take neither Spanish nor Calculus, and all others can take logic \\
\hline Comp- & $\mathrm{n}$ & One student can take neither Spanish nor Calculus, and all others cannot take logic \\
\hline False & $\mathrm{n}$ & All students can take Spanish and all students can take Calculus \\
\hline
\end{tabular}

The critical test sentences were variants of the stimuli 'Exactly one student can/cannot take Spanish or Calculus'

We asked participants if and to what extent they would infer a given candidate inference on a scale from 0 to $100 \%$, with $0 \%$ representing that a statement did not follow and $100 \%$ that it definitely followed.

For each scenario a context was given. Figure 1 shows an example trial that participants saw with sentence (3) in the TRUE condition. Table 2 shows an example item for each condition. For the sake of comparability, we here use the same statements across conditions whereas the actual items mentioned different classes. All context sentences and items can be found in Appendix A. ${ }^{15}$

In the TRUE condition, participants were expected to accept the given statement (i.e., give judgments close to 100\%), whereas in the FALSE condition they should clearly reject the statement (i.e., give judgments close to 0\%). Note that our TRUE

15 A link to the experiment is found under https://osf.io/2e4u $9 /$. 
condition is an entailment which should be judged as true no matter which reading participants adopt. In the critical FREE CHOICE conditions, participants judged the candidate inferences.

To ensure that the condition really served as a baseline for compatibility we had two versions of the COMPATIBLE conditions with identical sentences but different polarity in the second conjunct (e.g., COMPATIBLE+: One student can take either Biology or English, and all others can take Logic, vs. COMPATIBLE-: One student can take either Biology or English, and all others cannot take Logic). Therefore, if participants were to endorse one version accommodating contextual assumptions, they would not be able to use the same assumptions to endorse its negation. The two versions of this condition were distributed over two survey versions. Hence, a participant only saw one variant of the COMPATIBLE condition.

\subsection{Results and discussion}

The graph in Fig. 2 shows the mean percent of YES responses across conditions. As can be seen from the graph, the TRUE condition was rated highest, followed by the FREE CHOICE, COMPATIBLE, and FALSE conditions in both polarity conditions. The graph presents an average of the two versions of the compatible condition since respective judgments were similar across the two versions. ${ }^{16}$

We ran a series of mixed models to test statistical significance across conditions. First, we computed an omnibus model with sum coding of all factors. In particular, we included fixed effects for polarity, inference condition, and their interaction, as well as random slopes for participants and scenario. The results of the mixed model are summarized in Table 3.

The model revealed main effects for all comparisons across inference conditions. That is, the FREE CHOICE condition was rated significantly higher than the grand average $(p<.01)$, while the COMPATIBLE and FALSE conditions were rated significantly lower ( $p<.0001$, respectively). There was a marginal main effect of polarity $(p=.08)$ and a significant interaction across polarity in the FALSE condition $(p<.01)$. Crucially, there was no significant interaction between polarity and the FREE CHOICE condition $(p=.35) .{ }^{17}$

Second, we ran two separate models to assess whether individual comparisons across inference conditions are significant in both polarity conditions. For these models we set the FREE CHOICE target condition as the reference level (treatment coding). The models for the two polarity conditions revealed that in each case the FREE CHOICE condition was rated significantly lower than the TRUE condition (POSITIVE: $p$ value $<.0001$; NEGATIVE: $p$ value $<.05$ ). Critically, the FREE CHOICE condition was rated

\footnotetext{
16 We ran separate mixed model analyses for the two versions of the experiment and found that the compatible conditions were rated significantly lower than the FREE CHOICE condition and higher than the false baseline condition in both versions. As in the overall model reported in Table 3, no interaction between polarity and the target FREE CHOICE condition was present.

17 The interaction across polarity in the FALSE condition arguably reflects the fact that the expected response is reversed compared to the other inference conditions and participants might have been more likely to make mistakes with negation.
} 


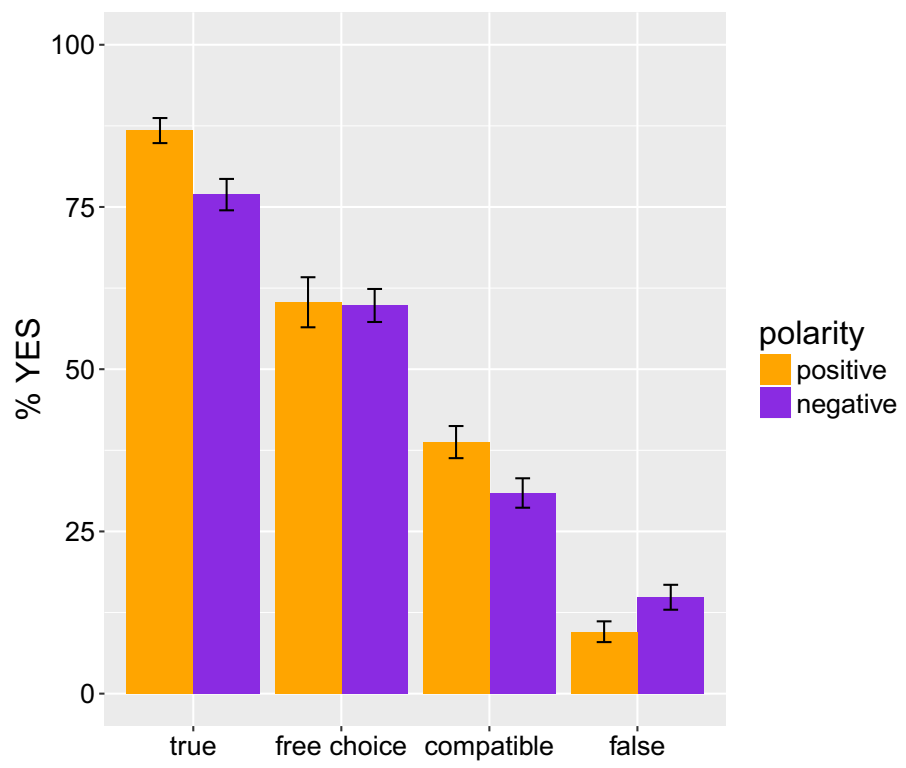

Fig. $2 \%$ YES by polarity (POSITIVE vs. NEGATIVE) and inference condition (TRUE, FREE CHOICE, COMPATIBLE, and FALSE) in Experiment 1. The rate of endorsement reflects the degree to which a candidate inference follows. Error bars represent SEM

Table 3 Results of mixed-effects model with sum coding of polarity and inference condition (Experiment 1)

\begin{tabular}{lrrrl}
\hline & Estimate & SE & $t$ value & $p$ value \\
\hline (Intercept) & 47.02 & 2.03 & 23.15 & \\
FREE CHOICE & 12.95 & 1.86 & 6.98 & 0.004 \\
COMPATIBLE & -12.45 & 1.43 & -8.73 & 0.0001 \\
FALSE & -35.07 & 1.44 & -24.29 & 0.0001 \\
Polarity & 1.55 & 0.88 & 1.77 & 0.083 \\
FREE CHOICE: polarity & -1.31 & 1.40 & -0.94 & 0.350 \\
COMPATIBLE: polarity & 2.32 & 1.41 & 1.65 & 0.100 \\
FALSE: polarity & -4.23 & 1.41 & -3.00 & 0.003 \\
\hline
\end{tabular}

higher than both the COMPATIBLE and FALSE baseline conditions in the POSITIVE and NEGATIVE cases (all $p$ values $<.0001$ ).

Overall, these results suggest that the POSITIVE variant (3) has the ALL-OTHERSDUAL-PROHIBITION reading, as predicted by the standard implicature approach: the FREE CHOICE condition differed from both the COMPATIBLE and the FALSE condition. Crucially, we found parallel differences in the case of (4), suggesting that the free choice reading is also available in the NEGATIVE environment. Hence, the findings of Experiment 1 provide a challenge for the standard implicature approach. 
Yet there are two potential issues concerning the target and COMPATIBLE conditions in Experiment 1. First, in the paraphrase of the candidate inference in the target condition we used the quantifier all, e.g. One student can take neither Spanish nor Calculus, and all others can choose between the two. This may have encouraged an alternative interpretation of the inference in the second conjunct, according to which the students as a group have a choice between Spanish and Calculus, with some students who can take Spanish and some who can take Calculus, without each of them having free choice. ${ }^{18}$ Further, it could be argued that the third item (e.g., the class Logic) mentioned in the COMPATIBLE condition lacks contextual relevance and therefore participants gave lower ratings in that condition. Note that all different items were mentioned in the preceding contexts and that we found similar results across positive and negative variants of this condition. Nevertheless, the issue of contextual relevance is potentially problematic. Therefore, we ran a second experiment with a modified version of the target and COMPATIBLE conditions, in order to replicate our main findings.

\section{Experiment 2}

\subsection{Goals and predictions}

Experiment 1 found evidence for free choice readings in both positive and negative environments. However, as discussed in the previous section, there are two potential issues with the statements used in Experiment 1. On the one hand, participants may have interpreted the candidate inference in the target FREE CHOICE conditions differently than intended, and on the other hand, the candidate inference used in the COMPATIBLE condition may have differed from that in the target conditions in terms of relevance, as it was about a third class not mentioned in the statement. For this reason, we modified the wording of the target and COMPATIBLE conditions in Experiment 2, seeking to replicate the results of Experiment 1. Specifically, we replaced the quantifier all in the second conjunct of the target inference with each and a partitive construction (e.g., One student can take neither Spanish nor Calculus and each of the others can choose between the two). This was done in order to ensure that the candidate inferences are interpreted with the intended ALL-OTHERS-DUAL-PROHIBITION and ALL-OTHERS-FREE-CHOICE readings. Further, the COMPATIBLE condition in Experiment 2 was refined to only talk about the classes mentioned in the statement. ${ }^{19} \mathrm{We}$ are confident, therefore, that the new COMPATIBLE condition provides a more solid baseline. All other items remained exactly the same as in Experiment 1.

\footnotetext{
18 Thanks to Angelika Kratzer (p.c.) for discussion on this.

19 Note further that the candidate inference, as in Experiment 1, is compatible with both the literal and local readings discussed above.
} 
Table 4 Example stimuli for Experiment 2

\begin{tabular}{|c|c|c|}
\hline Inference & Polarity & Candidate inference \\
\hline True & $\mathrm{p}$ & Only one student can take Spanish or Calculus \\
\hline $\mathrm{Fc}$ & $\mathrm{p}$ & $\begin{array}{l}\text { One student can choose between Spanish and Calculus, and each of the others can } \\
\text { take neither one }\end{array}$ \\
\hline Comp & $\mathrm{p}$ & $\begin{array}{l}\text { Some students can take neither Spanish nor Calculus, and some other can take } \\
\text { only one of the two }\end{array}$ \\
\hline False & $\mathrm{p}$ & No student can take Spanish and no student can take Calculus \\
\hline True & $\mathrm{n}$ & Only one student cannot take Spanish or Calculus \\
\hline $\mathrm{Fc}$ & $\mathrm{n}$ & $\begin{array}{l}\text { One student can take neither Spanish nor Calculus, and each of the others can } \\
\text { choose between the two }\end{array}$ \\
\hline Comp & $\mathrm{n}$ & $\begin{array}{l}\text { Some students can choose between Spanish and Calculus, and some other can take } \\
\text { only one of the two }\end{array}$ \\
\hline False & $\mathrm{n}$ & All students can take Spanish and all students can take Calculus \\
\hline
\end{tabular}

The critical test sentences were variants of the statement 'Exactly one student can/can't take Spanish or Calculus'

\subsection{Methods}

\subsubsection{Participants}

We recruited another set of 30 Participants with U.S. IP addresses via Amazon's Mechanical Turk and screened them for native language. They received 80 cents for participation in the study. All participants indicated their native language to be English (21 male, 9 female, mean age 30.8).

\subsubsection{Materials}

We used the test sentences and scenarios of Experiment 1 in Experiment 2. The main differences in Experiment 2 were that the target condition contained the quantifier each instead of all (POSITIVE: One student can choose between Spanish and Calculus, and each of the others can take neither one; and NEGATIVE: One student can take neither Spanish nor Calculus, and each of the others can choose between the two). Further, the COMPATIBLE condition was refined so that it was only about the two classes mentioned in the statement. The new candidate inference used in this condition was Some student can take neither Spanish nor Calculus, and some other can take only one of the two classes in the POSITIVE case, and Some student can choose between Spanish and Calculus, and some other can take only one of the two classes in the NEGATIVE one.

Table 4 shows an example item for each condition. All items are found in Appendix B. ${ }^{20}$

20 A link to the experiment is found under https://osf.io/uqwka/. 


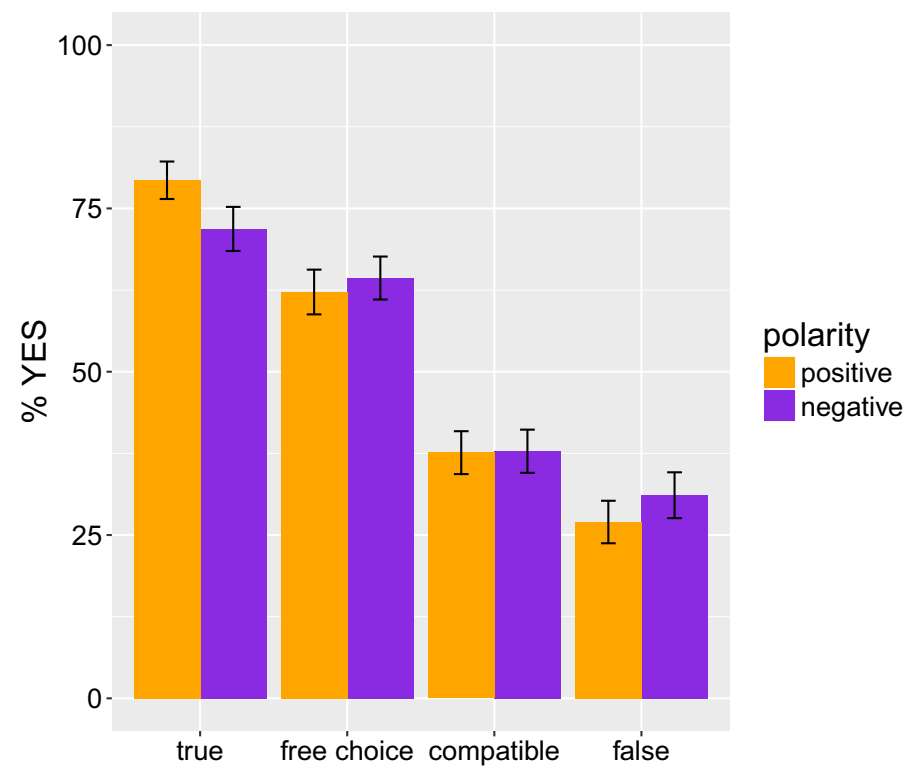

Fig. $3 \%$ YES by polarity (POSITIVE vs. NEGATIVE) and inference condition (TRUE, FREE CHOICE, COMPATIBLE, and FALSE) in Experiment 2. The rate of endorsement reflects the degree to which a candidate inference follows. Error bars represent SEM

Table 5 Results of mixed-effects model with sum coding of polarity and inference condition (Experiment 2)

\begin{tabular}{lccrr}
\hline & Estimate & SE & $t$ value & $p$ value \\
\hline (Intercept) & 52.53 & 3.35 & 15.69 & \\
FREE CHOICE & 10.65 & 1.77 & 6.03 & 0.0001 \\
COMPATIBLE & -12.29 & 1.71 & -7.17 & 0.0001 \\
FALSE & -20.15 & 1.72 & -11.74 & 0.0001 \\
Polarity & 0.15 & 1.36 & 0.11 & 0.9148 \\
FREE CHOICE: polarity & -1.15 & 1.71 & -0.67 & 0.5022 \\
COMPATIBLE: polarity & -0.19 & 1.71 & -0.11 & 0.9107 \\
FALSE: polarity & -2.04 & 1.72 & -1.19 & 0.2352 \\
\hline
\end{tabular}

\subsection{Results and discussion}

Figure 3 shows the mean percent of YES responses in Experiment 2.

We again computed a mixed-effects model with polarity, inference condition, and random slopes for participants and items for the data of Experiment 2. The model revealed main effects for all comparisons, with the FREE CHOICE condition being rated significantly higher than the grand average and the compatible and false conditions significantly lower (all $p$ values $<.0001$ ), as displayed in Table 5. There was no significant effect of polarity and there were no significant interactions across condi- 
tions. Further, two separate models with treatment coding were computed. The FREE CHOICE condition was rated significantly lower than the TRUE condition in the POSITIVE case $(p$ value $<.05)$, while judgments did not differ significantly in the NEGATIVE case $(p$ value $=.13)$. Crucially, the FREE CHOICE condition was rated higher than both the COMPATIBLE and FALSE baseline conditions in the POSITIVE ( $p$ values $<.01$ and .001, respectively) and NEGATIVE cases ( $p$ values $<.01$ and .001 , respectively).

These findings again indicate that both POSITIVE and NEGATIVE variants have a free choice reading. Crucially, we found parallel differences across inference conditions in both polarity conditions, replicating the findings of Experiment 1. In Experiment 2, we forced the intended free choice readings by using the quantifier each and a partitive construction. Furthermore, our new candidate inference in the COMPATIBLE condition was arguably as relevant as the corresponding one in the target conditions. The fact that participants distinguished the new versions of the target and COMPATIBLE conditions provides evidence therefore that both the ALL-OTHERS-DUAL-PROHIBITION and the ALL-OTHERS-FREE-CHOICE readings exist.

Overall, the results of Experiments 1 and 2 indicate that sentences of the type (9a) have the target ALL-OTHERS-DUAL-PROHIBITION reading, as predicted by the standard implicature approach. Participants showed the same pattern of responses in the case of (10a), suggesting that they accessed the ALL-OTHERS-FREE-CHOICE reading to a similar extent. Taken together, we take our findings to provide a clear challenge for the standard implicature approach. ${ }^{21}$

\section{Discussion: beyond the standard implicature approach}

As laid out above, our results provide a challenge for the standard implicature approach. This is because the standard approach does not predict the ALL-OTHERS-FREE-CHOICE reading. But, in both Experiment 1 and 2, we found that participants were more likely to endorse ALL-OTHERS-DUAL-PROHIBITION reading than a FALSE control and a statement that was merely compatible with the target sentence. Further, we found corresponding differences across conditions in the negative condition version, which probes the ALLOTHERS-FREE-CHOICE reading.

In this section, we sketch two possible strategies for addressing the challenge. The first is based on a more recent implicature account (Bar-Lev and Fox 2017; Bar-Lev 2018), based on the notion of innocent inclusion. The second involves tweaking the meaning of modified numerals with exactly. As we point out, both have substantial problems. In the next section, we turn to a third account that abandons the idea that free choice is an implicature.

\footnotetext{
21 In Experiment 2, simple baseline tests did not reveal a difference between the FREE CHOICE and TRUE condition for negative variants, but note that there was no interaction in the omnibus model.
} 


\subsection{The inclusion-based implicature account}

\subsubsection{The account}

The first attempt involves exploiting a different implicature algorithm, proposed recently by Bar-Lev and Fox (2017), Bar-Lev (2018) (see also Santorio 2018 for a similar proposal for handling similar phenomena in conditional antecedents). Like standard implicature accounts, Bar-Lev and Fox's algorithm works by conjoining with the assertion the negation of innocently excludable alternatives. In addition, it also conjoins with the assertion - 'includes' — a subset of other non-negated alternatives. Overall, this account works by supplementing the basic exclusion algorithm for scalar implicature with an extra step that further strengthens the sentence. The new algorithm requires a definition of innocently includable alternatives as follows: innocently includable alternatives are those that are in all maximal subsets of alternatives that can be conjoined consistently with the assertion and with the negation of innocently excludable alternatives. Based on (39), the definition of EXH is then straightforward: EXH conjoins the prejacent with all the innocently includable alternatives and the negation of all innocently excludable ones.

$$
\begin{aligned}
& \llbracket \operatorname{EXH} \rrbracket(C)(p)(w)= \\
& p_{w} \wedge \forall q \in \operatorname{IE}(p, C)\left[\neg q_{w}\right] \wedge \forall r \in \mathbb{I}(p, C)\left[r_{w}\right]
\end{aligned}
$$

To illustrate how EXH works, let's consider again (1a). Assume the sentence is now parsed as involving one occurrence of EXH, as in (41). We assume that the alternatives are the same as above in (24), repeated here in (42).

EXH[Emma can take Spanish or Calculus]

$\left\{\begin{array}{lr}\text { Emma can take Spanish or Calculus } & \nabla(\mathrm{S} e \vee \mathrm{C} e) \\ \text { Emma can take Spanish } & \diamond \mathrm{Se} \\ \text { Emma can take Calculus } & \nabla \mathrm{C} e \\ \text { Emma can take Spanish and Calculus } & \nabla(\mathrm{S} e \wedge \mathrm{C} e)\end{array}\right\}$

As before, the only innocently excludable alternative is $\diamond(\mathrm{Se} \wedge \mathrm{Ce})$. But now we have a further way of strengthening the basic meaning of the assertion, i.e. running the inclusion algorithm. As Bar-Lev and Fox (2017) show, there is only one subset of includable alternatives, $\{\diamond(\mathrm{S} e \vee \mathrm{C} e), \diamond \mathrm{S} e, \diamond \mathrm{C} e\}$. Thus we can include them all and obtain the free choice meaning of the sentence.

$$
\begin{aligned}
& \text { [EXH[Emma can take Spanish or Calculus }]] \rrbracket= \\
& \diamond(\mathrm{S} e \vee \mathrm{C} e) \wedge \neg \diamond(\mathrm{S} e \wedge \mathrm{C} e) \wedge \diamond \mathrm{S} e \wedge \diamond \mathrm{C} e
\end{aligned}
$$

Exclusion and inclusion accounts make identical predictions for basic cases of free choice. But they diverge for more complex sentences. In particular, Chemla (2009) has found that free choice effects arise robustly when a clause involving disjunction scoping under a modal appears embedded under quantifiers. For an example, consider (44). 
(44) Every girl can take Spanish or Calculus.

$\forall x \diamond(\mathrm{S} x \vee \mathrm{C} x)$

$\rightsquigarrow$ Every girl can choose between Spanish and Calculus

$\forall x(\diamond \mathrm{S} x \wedge \diamond \mathrm{C} x)$

Chemla finds that (44) has a reading suggesting that every girl can choose to take Spanish and can choose to take Calculus. Similarly, (45) has a reading suggesting that every girl has the option of avoiding Spanish as well as the option of avoiding Calculus. $^{22}$

$$
\begin{array}{lr}
\text { No girl has to take both Spanish and Calculus } & \neg \exists x \square(\mathrm{S} x \wedge \mathrm{C} x) \\
\rightsquigarrow \quad \text { Every girl can choose between avoiding Spanish and avoiding } \\
\text { Calculus } & \neg \exists x \square \mathrm{S} x \wedge \neg \exists x \square \mathrm{C} x
\end{array}
$$

(44) is predicted by exclusion approaches (together with the assumption that implicatures can be computed locally; see Chemla 2009 for discussion), but (45) is not. Bar-Lev and Fox (2017) use this as an empirical argument against exclusion accounts, and for inclusion accounts. ${ }^{23}$

Let us now turn to the non-monotonic cases that are our targets in this paper. As in the case of (45), the predictions of the two accounts diverge. Consider again (9a) and (10a).

(9a) Exactly one girl can take Spanish or Calculus.

$\rightsquigarrow$ One girl can choose between the two and each of the others can take neither of them

ALL-OTHERS-DUAL-PROHIBITION

(10a) Exactly one girl cannot take Spanish or Calculus.

$\rightsquigarrow$ One girl can take neither of the two and each of the others can choose between them

ALL-OTHERS-FREE-CHOICE

We saw that the exclusion account predicts the free choice reading of (3), but not that of (4). Let us now consider the predictions of the inclusion account. Crucially, we need to make assumptions about the set of alternatives in play. ${ }^{24}$ We assume that exactly one is replaceable with some and that the negation can be deleted. ${ }^{25}$

EXH[Exactly one girl can take Spanish or Calculus]

$$
\left\{\begin{array}{l}
\exists x^{|x|=1}[\diamond(\mathrm{S} x \vee \mathrm{C} x)] \\
\exists x^{|x|=1}[\diamond(\mathrm{S} x)] \\
\exists x^{|x|=1}[\diamond(\mathrm{C} x)] \\
\exists x^{|x|=1}[\diamond(\mathrm{S} x \wedge \mathrm{C} x)] \\
\exists x[\diamond(\mathrm{S} x \vee \mathrm{C} x)] \\
\exists x[\diamond(\mathrm{S} x)] \\
\exists x[\diamond(\mathrm{C} x)] \\
\exists x[\diamond(\mathrm{S} x \wedge \mathrm{C} x)]
\end{array}\right\}
$$

\footnotetext{
22 To better see the similarity between the two cases consider the equivalence between $\neg \exists x \square(S x \wedge C x)$ and $\forall x \diamond(\neg S x \vee \neg C x)$, and note that the free choice inference in (45) is equivalent to $\forall x(\diamond \neg S x \wedge \diamond \neg C x)$.

23 See Bar-Lev (2018) for other arguments for the inclusion account.

24 It's easy to check that these assumptions would not have made a difference for the exclusion case.

25 Note that this follows naturally from a theory of alternatives like Katzir (2007).
} 
Given this bigger set of alternatives, we can show that only the conjunctive alternatives $\exists x^{|x|=1}[\diamond(\mathrm{S} x \wedge \mathrm{C} x)]$ and $\exists x[\diamond(\mathrm{S} x \wedge \mathrm{C} x)]$ are excludable. All the other alternatives are includable. In particular, crucially for us, $\exists x^{|x|=1}[\diamond(\mathrm{S} x)]$ and $\exists x^{|x|=1}[\diamond(C x)]$ are includable. Together with the assertion, these alternatives directly entail the ALLOTHERS-DUAL-PROHIBITION reading. The reasoning is familiar from our discussion above. If there is exactly one student who can take Spanish or Calculus, exactly one who can take Spanish, and exactly one who can take Calculus, then one and the same student has to witness all three of these conditions. It follows that this one student has free choice and each of the other students is subject to dual prohibition.

Consider now the negative case in (48), assuming that we have the alternatives in (49). Notice that the alternative set includes the some-alternatives without negation (Some student can take Spanish, Some student can take Calculus). This assumption is crucial to get the prediction we want, as we will point out soon. (By contrast, further adding the exactly one-alternatives without negation and the some-alternatives with negation makes no difference.)

$$
\text { EXH[Exactly one girl cannot take Spanish or Calculus] }
$$

$$
\left\{\begin{array}{l}
\exists x^{|x|=1}[\neg \vee(\mathrm{S} x \vee \mathrm{C} x)] \\
\exists x^{|x|=1}[\neg \vee(\mathrm{S} x)] \\
\exists x^{|x|=1}[\neg \vee(\mathrm{C} x)] \\
\exists x^{|x|=1}[\neg \vee(\mathrm{S} x \wedge \mathrm{C} x)] \\
\exists x[\diamond(\mathrm{S} x \vee \mathrm{C} x)] \\
\exists x[\diamond(\mathrm{S} x)] \\
\exists x[\diamond(\mathrm{C} x)] \\
\exists x[\diamond(\mathrm{S} x \wedge \mathrm{C} x)]
\end{array}\right\}
$$

The only excludable alternatives are the conjunctive ones $\exists x^{|x|=1}[\neg \diamond(\mathrm{S} x \wedge \mathrm{C} x)]$ and $\exists x[\diamond(\mathrm{S} x \wedge \mathrm{C} x)]$. The includable ones are again all the others, giving rise to the reading we want. It's useful to visualize alternatives in a diagram (see Fig. 4). The innocently excludable alternatives are in gray, the innocently includable ones are underlined. The dotted ellipses represent maximal sets of innocently excludable alternatives. (We represent only some of the alternatives to avoid clutter.)

Let us highlight how the inclusion algorithm predicts the ALL-OTHERS-FREECHOICE reading. Crucially, given the alternative set in (49), the alternatives $\exists x^{|x|=1}[\neg \diamond \mathrm{S} x]$ and $\exists x^{|x|=1}[\neg \diamond \mathrm{S} x]$ are included. These alternatives say, respectively, that exactly one girl cannot take Spanish and exactly one girl cannot take Calculus. Given the proposition asserted, it must be that there is exactly one girl who is subjected to all these prohibitions. It follows that all the others are allowed to take Spanish and allowed to take Calculus.

Let us also highlight that the reason why the key alternatives $\exists x^{|x|=1}[\neg \diamond S x]$ and $\exists x^{|x|=1}[\neg \diamond \mathrm{S} x]$ are included is that the existential alternatives $\exists x[\diamond \mathrm{S} x]$ and $\exists x[\diamond C x]$ are in the alternative set. Recall that an alternative is innocently excludable only if its exclusion doesn't force the truth of other alternatives in the alternative set. It turns out that, if we try to negate one of $\exists x^{|x|=1}[\neg \diamond S x]$ and $\exists x^{|x|=1}[\neg \diamond S x]$, we are, in 


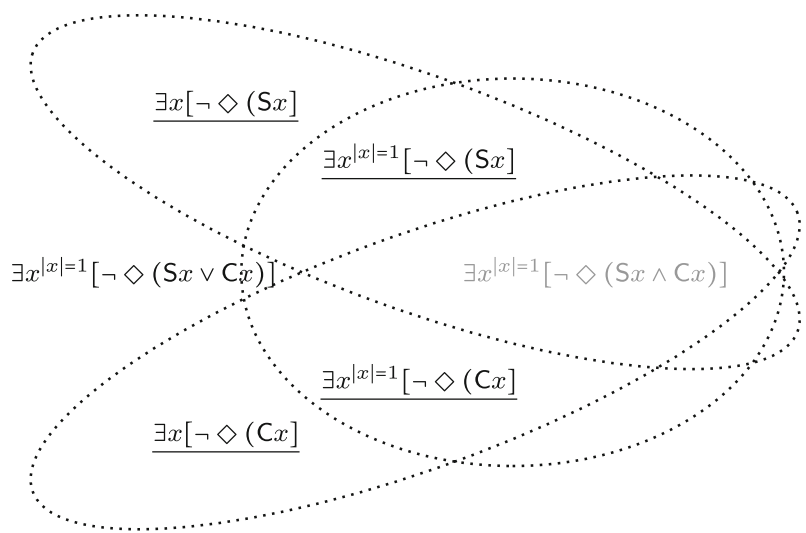

Fig. 4 Innocently excludable and innocently includable alternatives for (4): Exactly one student cannot take Spanish or Calculus

fact, forced to include one of the existential alternatives. ${ }^{26}$ As a result, the former alternatives are not innocently excludable. This makes them available for inclusion in the subsequent stage of the algorithm.

In summary: the inclusion-based account (given some assumptions about alternatives) can derive both readings. Hence, as long as we restrict consideration to (9a) and (10a), our results provide another argument for this account.

\subsubsection{Open problem: beyond exactly one}

While the inclusion approach nicely accounts for our results, a problem arises as soon as we move from to similar sentences involving a modified numeral exactly $n$, with $n$ higher than $1 .{ }^{27}$ Consider the following variant of (10a).

Exactly two girls cannot take Spanish or Calculus.

While we have not tested (50) experimentally, our intuitions converge on the judgment that it behaves exactly like (10a): it gives rise to a similar ALL-OTHERS-FREE-CHOICE reading (in this case, this reading amounts to the proposition that everyone aside from the two girls is allowed to take Spanish and is allowed to take Calculus). If this intuition were indeed correct, it would give rise to a potential problem. If we assume that exactly one is among the alternatives to exactly two (as e.g. Katzir's theory predicts, at least

\footnotetext{
26 For illustration: suppose that we negate $\exists x^{|x|=1}[\neg \diamond S x]$, i.e. we negate that exactly one student is not allowed to take Spanish. This means that either no students are not allowed to take Spanish, or more than one student is not allowed to take Spanish. The first disjunct is ruled out by the truth-conditional content of the sentence asserted (which establishes that exactly one student is subject to dual prohibition, and hence among other things is not allowed to take Spanish). It follows that more than one student is allowed to take Spanish. But again, the assertion tells us that exactly one student is subject to dual prohibition. It follows that there has to be some student who is not allowed to take Spanish, but is allowed to take Calculus (otherwise more than one student would be subject to dual prohibition). So it follows from the negation of the alternative that some student is allowed to take Calculus. But this is another alternative in the alternative set. Hence $\exists x^{|x|=1}[\neg \diamond S x]$ is not excludable, since negating it entails the truth of another alternative.

27 Thanks to Moshe Bar-Lev and Danny Fox for discussion on this point.
} 
as a possibility), then the derivation of the ALL-OTHERS-FREE-CHOICE effect for (50) should be blocked.

Let us explain briefly why. Recall that, in our derivation of the ALL-OTHERS-FREECHOICE effect for (4), it was crucial that the two alternatives Exactly one girl cannot take Spanish and Exactly one girl cannot take Calculus were includable. Similarly, to derive free choice for (50) we would need to include Exactly two girls cannot take Spanish and Exactly two girls cannot take Calculus. But these alternatives are not includable if their exactly one-counterparts are in the alternative sets. The reason is, pretty simply, that alternatives like the ones in (51) are mutually incompatible:

a. Exactly one student took Calculus.

b. Exactly two students took Calculus.

Innocently includable alternatives are the ones that are part of all the maximal sets of alternatives that can be consistently conjoined with the assertion. But the alternatives in (51) can never be in such a set together; hence neither turns out to be innocently includable. Of course, one could simply stipulate that a modified numeral of the form exactly $n$ can never be replaced by a modified numeral of the form exactly $n^{\prime}$ for the purposes of alternative generation. But to genuinely solve the issue, one should provide a principled account of this constraint. It is unclear to us that such account can be provided. In sum, at the current state of research, it looks like the approach based on innocent inclusion has to rely on a stipulation to get the right results beyond the exactly one case.

\subsection{A different meaning for modified numerals}

\subsubsection{The account}

The assumption up to now was that exactly $n$ has a generalized quantifier meaning as in $(52)^{28}$ :

$$
\text { 【exactly } \mathrm{n} \rrbracket=\lambda P \lambda Q[|P \cap Q|=n]
$$

This has however been criticised in the literature, notably by Landman (1998) (see also Spector 2014; Bar-Lev 2018), based on certain readings involving sentences with exactly, to which we shall briefly come back below. Based on these suggestions in the literature, we consider a different hypothesis about exactly $n$, which would essentially treat it as a reflection of EXH. In other words, and at a first approximation, the meaning of a sentence with exactly $n$ would be equivalent to the same sentence with the bare numeral $n$, but obligatorily exhaustified. Thus, our cases in (9a) and (10a) would become (53) and (54).

$$
\text { EXH[one student can take Spanish or Calculus] }
$$

\footnotetext{
28 Or, in the notation we used above:
}

(i) $\quad$ exactly $\mathrm{n} \rrbracket=\lambda P \lambda Q\left[\exists x^{|x|=n}[A x \wedge B x]\right]$ 
EXH[one student cannot take Spanish or Calculus]

As we show now, given this hypothesis about exactly and the LFs above, we can account for both the ALL-OTHERS-FREE-CHOICE and the ALL-OTHERS-DUAL-PROHIBITION readings. To illustrate, let's start from (54) and assume a simple exclusion-based EXH and an at-least meaning for numerals. The alternatives which EXH quantifies over in (54) include the following (where one is replaced with two, etc.):

$$
\left\{\begin{array}{l}
\exists x[|x| \geq 1 \wedge \neg \diamond(\mathrm{S} x \vee \mathrm{C} x)] \\
\exists x[|x| \geq 1 \wedge \neg \diamond(\mathrm{S} x)] \\
\exists x[|x| \geq 1 \wedge \neg \nabla(\mathrm{C} x)] \\
\exists x[|x| \geq 1 \wedge \neg \nabla(\mathrm{S} x \wedge \mathrm{C} x)] \\
\exists x[|x| \geq 2 \wedge \neg \nabla(\mathrm{S} x \vee \mathrm{C} x)] \\
\exists x[|x| \geq 2 \wedge \neg \nabla(\mathrm{S} x)] \\
\exists x[|x| \geq 2 \wedge \neg \diamond(\mathrm{C} x)] \\
\exists x[|x| \geq 2 \wedge \neg \nabla(\mathrm{S} x \wedge \mathrm{C} x)]
\end{array}\right\}
$$

The excludable alternatives include $\exists x[|x| \geq 2 \wedge \neg \vee(\mathrm{S} x)]$ and $\exists x[|x| \geq 2 \wedge \neg \vee(C x)]$. Their exclusion leads to the following: at least one student cannot take Spanish and cannot take Calculus, and no more than one cannot take Spanish, and no more than one cannot take Calculus. The first part guarantees the one dual prohibition, while the two excluded alternatives entail that each of the others can take Spanish and can take Calculus, i.e. has free choice.

$$
\exists x[|x| \geq 1 \wedge \neg \vee(\mathrm{S} x \vee C x)] \wedge \neg \exists x[|x| \geq 2 \wedge \neg \diamond(\mathrm{S} x)] \wedge \neg \exists x[|x| \geq 2 \wedge \neg \diamond(\mathrm{C} x)]
$$

The positive case can also be captured, though it requires postulating two EXH in the structure, as in (57).

\section{EXH[one student EXH[cannot take Spanish or Calculus]]}

The alternatives are the following:

$$
\left\{\begin{array}{ll}
\exists x[|x| \geq 1 \wedge \llbracket \mathrm{EXH} \rrbracket(\diamond(\mathrm{S} x \vee \mathrm{C} x))] & =\exists x[|x| \geq 1 \wedge \diamond(\mathrm{S} x \vee \mathrm{C} x) \wedge \neg \diamond(\mathrm{S} x \wedge \mathrm{C} x)] \\
\exists x[|x| \geq 1 \wedge \llbracket \mathrm{EXH} \rrbracket(\diamond(\mathrm{S} x)] & =\exists x[|x| \geq 1 \wedge \diamond(\mathrm{S} x \wedge \neg \diamond \mathrm{C} x)] \\
\exists x[|x| \geq 1 \wedge \llbracket \mathrm{EXH} \rrbracket(\diamond(\mathrm{C} x)] & =\exists x[|x| \geq 1 \wedge \diamond(\mathrm{C} x \wedge \neg \diamond \mathrm{S} x)] \\
\exists x[|x| \geq 1 \wedge \llbracket \mathrm{EXH} \rrbracket(\diamond(\mathrm{S} x \wedge \mathrm{C} x)] & =\exists x[|x| \geq 1 \wedge \diamond(\mathrm{C} x \wedge \mathrm{S} x)] \\
\exists x[|x| \geq 2 \wedge \llbracket \mathrm{EXH} \rrbracket(\diamond(\mathrm{S} x \vee \mathrm{C} x))] & =\exists x[|x| \geq 2 \wedge \diamond(\mathrm{S} x \vee \mathrm{C} x) \wedge \neg \diamond(\mathrm{S} x \wedge \mathrm{C} x)] \\
\exists x[|x| \geq 2 \wedge \llbracket \mathrm{EXH} \rrbracket(\diamond(\mathrm{S} x)] & =\exists x[|x| \geq 2 \wedge \diamond(\mathrm{S} x \wedge \neg \diamond \mathrm{C} x)] \\
\exists x[|x| \geq 2 \wedge \llbracket \mathrm{EXH} \rrbracket(\diamond(\mathrm{C} x)] & =\exists x[|x| \geq 2 \wedge \diamond(\mathrm{C} x \wedge \neg \nabla \mathrm{S} x)] \\
\exists x[|x| \geq 2 \wedge \llbracket \mathrm{EXH} \rrbracket(\diamond(\mathrm{S} x \wedge \mathrm{C} x)] & =\exists x[|x| \geq 2 \wedge \diamond(\mathrm{C} x \wedge \mathrm{S} x)]
\end{array}\right\}
$$

The crucial excludable ones are: $\exists x[|x| \geq 1 \wedge \llbracket \operatorname{EXH} \rrbracket(\diamond(\mathrm{S} x)], \exists x[|x| \geq 1 \wedge$ $\llbracket \mathrm{EXH} \rrbracket(\diamond(\mathrm{C} x)]$, and $\exists x[|x| \geq 2 \wedge \llbracket \mathrm{EXH} \rrbracket(\diamond(\mathrm{S} x \vee \mathrm{C} x))]$. Their negation, together with the prejacent, leads to the conjunction of the following:

$$
\begin{array}{ll}
\text { a. } & \exists x[|x| \geq 1 \wedge \diamond(\mathrm{S} x \vee \mathrm{C} x) \\
\text { b. } \neg \exists x[|x| \geq 1 \wedge \diamond(\mathrm{C} x \wedge \neg \diamond \mathrm{S} x)] \\
\text { c. } \neg \exists x[|x| \geq 1 \wedge \diamond(\mathrm{C} x \wedge \neg \vee \mathrm{S} x)] \\
\text { d. } \neg \exists x[|x| \geq 2 \wedge \diamond(\mathrm{S} x \vee \mathrm{C} x) \wedge \neg \vee(\mathrm{S} x \wedge \mathrm{C} x)] \wedge \neg \exists x[|x| \geq 2 \wedge \diamond(\mathrm{C} x \wedge \mathrm{S} x)
\end{array}
$$


(59a-d) entails the ALL-OTHERS-DUAL-PROHIBITION reading. Informally, (59a) conveys that at least one student can do at least one of Spanish or Calculus. (59b) and $(59 \mathrm{c})$ entail that nobody can do only one of the two, and (59d) guarantees that no more than one can take at least one of Spanish and Calculus. These conditions altogether entail that there is only one student who can do Spanish and Calculus and, given that nobody can only do one of the two, this student must have free choice between the two. All of the others can't do either one, i.e. have dual prohibition. In sum, the hypothesis above, by postulating a non-standard meaning for modified numerals with exactly, can account for our results.

\subsubsection{Open issues}

One worry for this kind of account is that it concerns specifically sentences involving modified numerals of the form exactly $n$. While our experimental data only involves phrases of this sort, we conjecture that the issue will concern a much larger class of non-monotonic phrases, including phrases involving overt only or modifiers like just and between $n$ and $n^{\prime}$. Some relevant examples are given below.

(60) Only two students can/cannot take Spanish or Calculus.

(61) Just two students can/cannot take Spanish or Calculus.

(62) Between two and four students can/cannot take Spanish or Calculus.

Of course we have only tested the case of exactly one; the cases above should also be tested experimentally. But if our intuitions about them are right, it is unclear that the account described in this section can be extended to sentences like (60)-(62).

\subsection{Taking stock}

We have sketched two main options for amending the implicature approach in order to account for our results. Neither seems satisfactory at this stage. This doesn't preclude the possibility that further modifications of the implicature approach would work, but it shows that the challenge is nontrivial. Before moving to what seems a more promising strategy, we briefly outline the options for the implicature account as we see them at the moment.

The implicature theorist has four main parameters to manipulate: (i) the meaning of quantified phrases of the form exactly $n$; (ii) the alternatives used for exhaustification; (iii) the number and position of exhaustivity operators in the sentence; (iv) the algorithm for computing implicatures (innocent exclusion, innocent inclusion, or something else).

As we pointed out, the difficulty for accounts that revolve around manipulating (i) is that they might not be general enough. The behavior of free choice inferences under non-monotonic quantifiers deserves more extensive experimental investigation than we have done here. If (as seems plausible to us) the phenomenon we found generalizes to other quantifiers, this approach will lack generality. 
Options to manipulate (ii) and (iii) look more promising. One may find a set of alternatives and/or a parsing that predict the right outcome. Of course, it would be desirable if the choice of these alternatives and/or parsing could receive independent justification, beyond mere stipulation. ${ }^{29}$

Finally, a fourth option for the implicature theorist would be to refine even further the mechanism that generates implicatures (possibly in combination with one of the other options above). The risk behind this strategy, of course, is to produce overgeneration. In particular, the innocent inclusion algorithm already produces very strong meanings. Again, we leave attempts of this sort to future work.

\section{A semantic account}

\subsection{Free choice and homogeneity}

Recent literature on free choice includes a number of theories that predict the phenomenon on semantic grounds. While semantic accounts have existed since the beginning of the debate on free choice (see e.g. Zimmerman 2000), recent theories have made substantial empirical progress, since they predict dual prohibition alongside free choice. Accounts in this vein include, among others, Starr (2016), Aloni (2016), Willer (2017), Goldstein (2018), and Rothschild and Yablo (2018). For concreteness here we focus on the account in Goldstein (2018) and sketch its predictions in relation to our results. In particular, we focus on the version of Goldstein's theory based on alternative semantics. But as far as we can see, our points apply similarly to other accounts within the semantic approach to free choice.

29 A promising option pointed out to us by an anonymous reviewer is a parse involving an embedded and a global EXH, as in (i).

(i) $\operatorname{EXH}\left[[\text { exactly two students }]_{1}\left[\mathrm{EXH}\left[\operatorname{not}\left[t_{1}\right.\right.\right.\right.$ took Spanish or Calculus $\left.\left.\left.]\right]\right]\right]$

This parse, with the alternatives in (ii) for the outermost EXH, correctly gives rise to the ALL-OTHERS-FREECHOICE reading, for any $n$ in exactly $n$. This is because all of the alternatives in (ii) other than the prejacent are innocently excludable and the conjunction of their negation with the prejacent entails that two students cannot take either of the two classes and none of the others can take one but not the other, hence each of the others has free choice between them, as shown in (iii).

(ii)

$$
\left\{\begin{array}{l}
\exists x^{|x|=2}[\neg \diamond(\mathrm{S} x \vee \mathrm{C} x)] \\
\exists x^{|x|=2}[\neg \diamond \mathrm{S} x \wedge \diamond \mathrm{C} x] \\
\exists x^{|x|=2}[\neg \diamond \mathrm{C} x \wedge \diamond \mathrm{S} x] \\
\exists x[\neg \diamond \mathrm{S} x \wedge \diamond \mathrm{C} x] \\
\exists x[\neg \diamond \mathrm{C} x \wedge \diamond \mathrm{S} x]
\end{array}\right\}
$$

$$
\begin{aligned}
& \exists x^{|x|=2}[\neg \diamond(\mathrm{S} x \vee \mathrm{C} x)] \wedge \\
& \neg[\exists x|x|=2[\neg \diamond \mathrm{S} x \wedge \diamond \mathrm{C} x]] \wedge \neg\left[\exists x^{|x|=2}[\neg \diamond \mathrm{C} x \wedge \diamond \mathrm{S} x]\right] \wedge \neg \exists x[\neg \diamond \mathrm{S} x \wedge \diamond \mathrm{C} x] \wedge \neg \exists x[\neg \diamond \mathrm{C} x \wedge \diamond \mathrm{S} x] \\
& =\exists x^{|x|=2}[\neg \diamond(\mathrm{S} x \vee \mathrm{C} x)] \wedge \neg \exists x[\neg \diamond \mathrm{S} x \wedge \diamond \mathrm{C} x] \wedge \neg \exists x[\neg \diamond \mathrm{C} x \wedge \diamond \mathrm{S} x]
\end{aligned}
$$

Note that it is crucial here that the conjunctive alternative is pruned for the derivation to work. There is therefore a question as to why this should be necessary (but see Crnic et al. 2015, which, in relation to a similar case involving the inferences of disjunction in the scope of universal quantifiers, has proposed a constraint on pruning that would result in that effect). 
Goldstein's account is based on two main ingredients. First, he uses a strong meaning for a configuration like (63); in particular, the free choice effect is directly part of the truth-conditional meaning of the sentence. Second, Goldstein postulates that the modal triggers a homogeneity inference, which requires that either both disjuncts are possible or neither of them is. ${ }^{30}$

Let us spell out the account in some detail. Goldstein uses a trivalent semantics, on which all clauses are mapped to one of three truth values: true, false, and indeterminate (represented as '\#'). The semantics derives free choice via two main assumptions. First, in alternative semantics, disjunctive clauses introduce sets of alternatives: hence e.g. $A \vee B$ denotes a set containing the two propositions $\llbracket \mathrm{A} \rrbracket$ and $\llbracket \mathrm{B} \rrbracket$. Second, the lexical meaning of possibility modals involves a homogeneity requirement to the effect that the alternatives denoted by the prejacent should both be evaluated in the same way. For the moment we'll follow Goldstein in thinking of this requirement as a presupposition, though this is not essential.

These are Goldstein's lexical entries. Notice that ' $\odot$ ' is the object language possibility modal, which is defined by appealing to a metalanguage modal ' $\diamond$ '.

$$
\begin{array}{ll}
\text { a. } & \llbracket \mathrm{p} \rrbracket=\{\lambda w . p(w)=1\} \\
\text { b. } & \llbracket \neg A=W-\cup \llbracket \mathrm{A} \rrbracket \\
\text { c. } & \llbracket A \vee B \rrbracket=\llbracket \mathrm{A} \rrbracket \cup \llbracket \mathrm{B} \rrbracket \\
\text { d. } & \llbracket \odot A \rrbracket=\{\lambda w: \exists v \in\{0,1\} \forall \mathbf{A} \in A, \diamond \mathbf{A}(w)=v . \forall \mathbf{A} \in A, \diamond \mathbf{A}(w)=1\}
\end{array}
$$

Here is, schematically, how this system derives free choice. Take $\odot(A \vee B) . A \vee B$ denotes a set of two propositions, i.e. $\{\mathbf{A}, \mathbf{B}\}$. Given the lexical meaning of $\odot, \diamond(A \vee B)$ presupposes that $\diamond \mathbf{A}$ and $\diamond \mathbf{B}$ have the same truth value, and asserts that they are both true. Once we place the whole clause under negation, via the homogeneity condition we get that, whenever the sentence is defined and true, both $\diamond \mathbf{A}$ and $\diamond \mathbf{B}$ have to be false. This is because the presupposition requires that they are either both true or both false and the sentence asserts that it's false that they are both true. As a result, the semantics correctly predicts the free choice reading of (64) and the dual prohibition reading of (65).
a. Emma can take Spanish or Calculus.
b. $\rightsquigarrow$ Emma can take Spanish and she can take Calculus
a. Emma can't take Spanish or Calculus.
b. $\rightsquigarrow$ Emma cannot take Spanish and she cannot take Calculus

In summary: the basic meaning of the sentence, in combination with a homogeneity inference triggered by the modal, manages to capture the attested pattern involving free choice and dual prohibition for basic sentences.

\footnotetext{
30 Notice that we talk about a homogeneity inference, without deciding whether homogeneity is a presupposition, as traditionally assumed, or whether it is some other kind of phenomenon, as Križ (2015) has recently argued. More on this below.
} 


\subsection{Homogeneity projection}

To see how this account extends to our non-monotonic cases, we have to ask how homogeneity effects work in complex sentences that involve non-monotonic phrases-in other words, how homogeneity projects in sentences like (9a) and (10a). What projection behavior we predict for homogeneity triggers will depend on what account of homogeneity we endorse (see Križ and Chemla 2015 for discussion and experimental data on homogeneity projection with plural definites). Here we remain neutral as to what particular account of homogeneity one should adopt. Rather, we state in a precise way the particular formal property we need (roughly: universal projection of homogeneity under non-monotonic quantifiers), and then we sketch how all main accounts of the phenomenon predict it.

This is the result with respect to projection that we're going to need.

\section{Universal projection of truth (UPT)}

Let $Q x$ be a non-monotonic quantifier, and $S(x)_{h}$ an (open) sentence that triggers a homogeneity inference $h$. Then a sentence of the form $\left\ulcorner Q x\left[S(x)_{h}\right]\right\urcorner$ is defined and true only if, for all $x$ in the domain of $Q, h(x)$ is true.

To illustrate, consider again (9a), repeated below.

(9a) Exactly one girl can take Spanish or Calculus.

UPT requires that, for (3) to be true, the relevant homogeneity property has to hold for all the individuals in the domain of quantification. Given a Goldstein-style semantics for the modal, this means that each of the students has to either be allowed to take Spanish and allowed to take Calculus, or allowed to take neither. This immediately entails the ALL-OTHERS-FREE-CHOICE reading. Similarly, mutatis mutandis, for (10a) and the ALL-OTHERS-DUAL-PROHIBITION reading. ${ }^{31}$

As it turns out, UPT is predicted on most of the main existing approaches to homogeneity projection. In particular, UPT is predicted on approaches based on strong Kleene logic (see Goldstein 2018; Križ 2015 for discussion). On a strong Kleene approach, the projection of indeterminacy is handled in a supervaluational fashion. Roughly: we consider all ways to resolve indeterminate cases as true or as false. If all the resolutions agree on the assignment of truth value to the whole sentence, then the sentence is defined; otherwise it is undefined. For example, suppose that the students in my class have different requirements with respect to what class they can or cannot take. There are three students in the the class: Ann, Bill, and Carl. Assume that Ann can take Spanish and that she can take Calculus, Bill can only take Calculus, and Carl cannot take either. Then the predicate can take Spanish or Calculus yields True when applied to Ann, False to Carl, and Undefined to Bill. In this scenario, consider the following sentences:

\footnotetext{
31 Note that differently from what standardly happens in the literature on presupposition, we are stating the projection property in terms of a particular truth value (namely, True), rather than in terms of definedness. The reason is that, as highlighted by Križ (2015, chapter 1), for non-monotonic quantifiers the analog of UPT will not hold in all cases for falsity. We refer the reader to Križ's discussion for a full explanation of this point.
} 
(67) Some students can take Spanish or Calculus.

(68) All students can take Spanish or Calculus.

(69) Exactly one student can take Spanish or Calculus.

On the strong Kleene account, (67) is true in the scenario described. No matter how the Bill case is resolved, Ann's having free choice between the two classes is sufficient to make it true. Conversely, (68) is false in this scenario. No matter how the Bill case is resolved, Carl's not being allowed to take either of the classes is sufficient to make it false. Finally, (69) is undefined in the scenario. The reason is that, depending on how we resolve the Bill case, the quantified sentence can be true or false. If we turn the '\#' to True, then it is true that exactly two students bought the books, and if we turn it to False, it is false that exactly two students bought the books. Moreover, it's easy to see that the only outcome in which we will get a verdict of True for a sentence like (69) is a case in which the homogeneity condition is satisfied by all individuals in the domain. If homogeneity fails for even just one individual, then the sentence will turn out either false or undefined. This illustrates that UPT holds for sentences involving quantificational determiners like exactly two. ${ }^{32,33}$

In summary: In this section, we have investigated the predictions of a homogeneitybased account of free choice in the style of Goldstein (2018) for our examples. As we pointed out, the predictions depend on assumptions about the projection of homogeneity effects in complex sentences. On all the main approaches to homogeneity projection, however, we find that homogeneity inferences project universally under quantifiers like exactly one and exactly two. That is, a sentence of the form $\left\ulcorner Q x\left[S(x)_{h}\right]\right\urcorner$ (with $Q$ a non-monotonic quantifier) is true only if, for all $x$ in the domain of $Q, h(x)$ is true. This shows that a homogeneity-based theory of free choice can account for our results.

\subsection{Open issues}

Of course, the semantic account that we have sketched in this section leaves open a variety of issues as well; see e.g. Romoli and Santorio (2019), Marty and Romoli (2019) for discussion. Here we mention a couple. First, the approach in Goldstein (2018) does not extend in any obvious way to the so-called distributive inferences of the type in (70), which disappear under negation, as in (71).

(70) Emma is required to take Spanish or Calculus.

$\rightsquigarrow$ Emma can take Spanish and she can take Calculus

(71) Emma is not required to take Spanish or Calculus.

$\rightsquigarrow$ Emma is not required to take Spanish and she is not required to take Calculus

\footnotetext{
32 Notice also that the strong Kleene account makes a number of subtle and interesting predictions for other non-monotonic quantifiers. For example, it will not require that homogeneity projects universally for a quantifier like between three and five students. This might provide the opportunity for testing the predictions of this account in comparison to other accounts; we have to leave such tests to future work.

33 A similar point can be made for the ambiguity account by Križ and Spector (2017).
} 
Intuitively, the same pattern of readings we found with free choice and non-monotonic quantifiers can be reproduced here as well. For instance, (72) suggests that one student is required to take Spanish or Calculus and allowed to take either of the two, while all of the others are not required to take either of the two. It is not clear how to obtain the reading in (72) under this approach.

(72) Exactly one student is required to take Spanish or Calculus. $\rightsquigarrow$ One student is required to take Spanish or Calculus and allowed to take either of the two and each of the others are not required to take either of the two

Similarly, semantic accounts do not derive the so-called negative free choice inference of cases like (see Fox (2007) for discussion and Chemla (2009) for experimental evidence for this inference; see also Ciardelli et al. (2018) for a dissenting view on those data).

(73) Emma is not required to take Spanish and Calculus

$\rightsquigarrow$ Emma is not required to take Spanish and she is not required to take Calculus NEGATIVE FC

We can again modify the configuration in (73) by substituting a non-monotonic quantifier like in (74), extending the problem: (74) intuitively suggests that one student is not required to take Spanish and not required to take Calculus, while all of the others are required to take both. The first part of the inference, corresponding to negative free choice, is problematic for semantic accounts like that of Goldstein (2018).

(74) Exactly one student is not required to take Spanish and Calculus. $\rightsquigarrow$ One student is not required to take Spanish and is not required to take Calculus and all of the others are required to take both

In sum, the homogeneity approach can account for our results, but there are a variety of related cases which are problematic for this approach, as discussed in the literature. Here we have briefly shown that those cases extend to the scope of non-monotonic quantifiers, in a similar fashion to the configurations explored in this paper. ${ }^{34}$

\footnotetext{
34 Another open question for the homogeneity approach is what exactly is the nature of homogeneity and how it relates to other phenomena for which homogeneity has been invoked. In particular, there are various reasons to believe we should not conceive it of as a presupposition, as Goldstein (2018) and Rothschild and Yablo (2018) discuss, because homogeneity does not seem to project like presuppositions; (i) does not suggest that Emma can either take both or neither, which would correspond to the homogeneity inference projecting out of the antecedent of the conditional in the way in which a presupposition would.
}

(i) If Emma can take Spanish or Calculus, Sue will be happy.

ঋ Emma can either take both or she can take neither

We can of course conceive homogeneity not as a presupposition but as something else, assimilating it more with what has been proposed for definites (Križ 2015). This would require a detailed comparison between homogeneity projection for definites and projection of free choice effects. A first step in this direction could be to ask whether definites also require universal projection in cases analogous to our target sentences - that is, whether (iia) and (iiia) give rise to the readings in (iib) and (iiib), which correspond to our ALL-OTHERS-DUAL-PROHIBITION and ALL-OTHERS-FREE-CHOICE readings. 


\section{Conclusion}

Disjunctions in the scope of possibility modals give rise to a conjunctive inference, generally labeled 'free choice.' For example, Emma can take Spanish or Calculus suggests that she can 'choose' between the two. As is well known, this inference doesn't follow from standard semantics for modals and disjunction. To complicate things further, free choice tends to disappear under negation: Emma cannot take Spanish or Calculus doesn't merely suggests that she can't choose between the two, but rather that she can take neither. Free choice has been at the centre of attention in philosophy of language and semantics since the seventies. Theories of free choice not only have to tell us how it arises in positive contexts but also how it disappears in negative ones. In this paper we have looked at this problem, focusing on the predictions of a prominent approach in the literature which treats free choice as a scalar implicature. In particular, we investigated sentences which contain a non-monotonic modified numeral and embed a disjunction under a possibility modal, as in Exactly one student can(not) take Spanish or Calculus. We then reported on two experiments investigating the possible free choice readings of these sentences, using an inferential task. As we discussed, our results challenge the standard implicature approach, as the latter doesn't predict all the readings we find evidence for. We sketched two possible strategies to account for the data within an implicature framework. The first relies on a new algorithm for implicature computation and the second adopts a different meaning for modified numerals with exactly. Both have a variety of problems at this stage. We then turned to a more promising third solution, based on a homogeneity account of free choice. Regardless of the solution one chooses, we submit that non-monotonic quantifiers are an important test case for our understanding of free choice, implicature, and modified numerals.

Before concluding, let us point to similar configurations beyond free choice and non-monotonic quantifiers, the investigation of which would help with the choice points above. We mentioned cases not involving non-monotonic quantifiers in Sect. 5.2.2 above. Here we want to briefly discuss a few data points not involving free choice. Consider a simple disjunction first, like (75), and its negation in (76). The former is well known to give rise to the exclusivity inference that Emma didn't take both of the classes, which disappears under negation in the latter, which conveys that Emma didn't take either of the two.

Emma took Spanish or Calculus.

$\rightsquigarrow$ Emma didn't take both of the classes

EXCLUSIVITY

Footnote 34 continued

(ii) a. Exactly one student met the professors.

b. ? $\rightsquigarrow$ One student met all of the professors and each of the others didn't meet any of them

(iii) a. Exactly one student didn't meet the professors.

b. ?œ One student didn't meet any of the professors and each of the others met all of them

In sum, the open question for this account concerns the nature of the homogeneity involved with free choice and how it relates to presuppositions, on the one hand, and to the homogeneity invoked for definite plurals, on the other. 
Emma didn't take Spanish or Calculus

$\rightsquigarrow$ Emma didn't take either of the classes

NEITHER

The exclusivity inference in (75) is a paradigmatic case of implicature. The relevant question for us is what happens in the configurations with non-monotonic quantifiers we explored above. In particular, focusing on the negative case, the question is whether (77) give rise to what we can call an ALL-OTHERS-EXCLUSIVITY inference.

(77) Exactly one student didn't take Spanish or Calculus.

? $\rightsquigarrow$ One student didn't take either and each of the others took one or the other but not both

ALL-OTHERS-EXCLUSIVITY

To sharpen intuitions again, consider our usual model with three individuals: Ann, Bill, and Carl. This reading of (77) would convey that we are in a situation like the one described in (78).

\begin{tabular}{|c|c|c|}
\hline Ann & $\neg \mathrm{S} a \wedge \neg \mathrm{C} a$ & NEITHER \\
\hline Bill & $(\mathrm{S} b \vee \mathrm{C} b) \wedge \neg(\mathrm{S} b \wedge \mathrm{C} b)$ & EXCLUSIVITY \\
\hline Carl & $(\mathrm{S} c \vee \mathrm{C} c) \wedge \neg(\mathrm{S} c \wedge \mathrm{C} c)$ & EXCLUSIVITY \\
\hline
\end{tabular}

Similar data can be reproduced with the not-required implicature of modals like (79). Again, the configuration in (79) does not seem to suggest that each of the other students is allowed but not required to take Spanish (i.e., (80) seems silent about whether some other students are required to take Spanish).

Emma is allowed to take Spanish.

$\rightsquigarrow$ Emma is not required to take Spanish

(80) Exactly one student is not allowed to take Spanish.

$? \rightsquigarrow$ One student is not allowed to take Spanish and each of the others are allowed but not required

ALL-OTHERS-NOT-REQUIRED

While we do not have experimental data on (80), we don't think that (80) can have this reading. If this is correct, this lack of analogy would be another potential problem for the implicature approach: one should make sure that any modification intended to account for the free choice case does not extend and overgenerate in the case of simple disjunctions or simple modals.

Acknowledgements For very helpful discussion and suggestions we thank Maria Aloni, Amir Anvari, Moysh Bar-Lev, Danny Fox, Simon Goldstein, Nathan Klinedinst, Angelika Kratzer, Matt Mandelkern, Paul Marty, Philippe Schlenker, Benjamin Spector, Yasu Sudo, Malte Willer, and audiences at SALT28 MIT and in Amsterdam, Paris, London, and Berlin. This work was financially supported by the Deutsche Forschungsgemeinschaft (DFG) as part of the Xprag.de Initiative (BE 4348/4-1) and the Leverhulme Trust Grant RPG-2018-425. Experiment 1 of this paper was published in the Proceedings of SALT 28. The current paper goes substantially beyond the proceedings paper in terms of theoretical discussion and empirical findings. Author names are listed alphabetically.

Open Access This article is licensed under a Creative Commons Attribution 4.0 International License, which permits use, sharing, adaptation, distribution and reproduction in any medium or format, as long as you give appropriate credit to the original author(s) and the source, provide a link to the Creative Commons licence, 
and indicate if changes were made. The images or other third party material in this article are included in the article's Creative Commons licence, unless indicated otherwise in a credit line to the material. If material is not included in the article's Creative Commons licence and your intended use is not permitted by statutory regulation or exceeds the permitted use, you will need to obtain permission directly from the copyright holder. To view a copy of this licence, visit http://creativecommons.org/licenses/by/4.0/.

\section{References}

Aloni, M. 2016. FC disjunction in state-based semantics. Unpublished manuscript, UVA.

Alonso Ovalle, L. 2005. Disjunction in alternative semantics. PhD thesis, University of Massachusetts.

Bar-Lev, M. 2018. Free choice, homogeneity, and innocent inclusion. PhD thesis, MIT.

Bar-Lev, M., and D. Fox. 2017. Universal free choice and innocent inclusion. Proceedings of SALT 27, 95-115. Washington, DC: LSA.

Bassi, I., and M. Bar-Lev. 2016. A unified existential semantics for bare conditionals. Proceedings of Sinn und Bedeutung, vol. 21. https://semanticsarchive.net/Archive/DRjNjViN/index.html.

Breheny, R., N. Klinedinst, J. Romoli, and Y. Sudo. 2018. The symmetry problem: Current theories and prospects. Natural Language Semantics 26 (2): 85-110.

Chemla, E. 2009. Universal implicatures and free choice effects: Experimental data. Semantics and Pragmatics 2 (2): 1 .

Chemla, E. 2010. Similarity: Towards a unified account of scalar implicatures, free choice permission and presupposition projection. Unpublished manuscript, LSCP \& MIT.

Chemla, E., and L. Bott. 2014. Processing inferences at the semantics/pragmatics frontier: Disjunctions and free choice. Cognition 130 (3): 380-396.

Chemla, E., and B. Spector. 2011. Experimental evidence for embedded scalar implicatures. Journal of Semantics 28 (3): 359-400.

Chierchia, G. 2004. Scalar implicatures, polarity phenomena, and the syntax/pragmatics interface. In Structures and beyond: The cartography of syntactic structures, vol. 3, ed. A. Belletti, 39-103. Oxford: Oxford University Press.

Chierchia, G. 2013. Logic in grammar: Polarity, free choice, and intervention. Oxford: Oxford University Press.

Chierchia, G., D. Fox, and B. Spector. 2012. The grammatical view of scalar implicatures and the relationship between semantics and pragmatics. In Semantics: An international handbook of natural language meaning, vol. 3, ed. C. Maienborn, K. von Heusinger, and P. Portner. Berlin: Mouton de Gruyter.

Ciardelli, I., Z. Linmin, and L. Champollion. 2018. Two switches in the theory of counterfactuals: A study of truth conditionality and minimal change. Linguistic and Philosophy 41: 577-621.

Crnic, Luka L., E. Chemla, and D. Fox. 2015. Scalar implicatures of embedded disjunction. Natural Language Semantics 23: 271-305.

Fox, D. 2007. Free choice and the theory of scalar implicatures. In Presupposition and implicature in compositional semantics, ed. U. Sauerland, and P. Stateva, 71-120. London: Palgrave.

Fox, D., and B. Spector. 2018. Economy and embedded exhaustification. Natural Language Semantics 26 (1): $1-50$.

Franke, M. 2011. Quantity implicatures, exhaustive interpretation, and rational conversation. Semantics and Pragmatics 4 (1): 1-82.

Goldstein, S. 2018. Free choice and homogeneity. Manuscript, Lignan University (Hong Kong).

Gotzner, N., and J. Romoli. 2017. The scalar inferences of strong scalar terms under negative quantifiers and constraints on the theory of alternatives. Journal of Semantics 35 (1): 95-126.

Kamp, H. 1974. Free choice permission. Proceedings of the Aristotelian Society 74: 57-74.

Katzir, R. 2007. Structurally-defined alternatives. Linguistic and Philosophy 30 (6): 669-690.

Klinedinst, N. 2007. Plurality and possibility. PhD thesis, UCLA.

Kratzer, A., and J. Shimoyama. 2002. Indeterminate pronouns: The view from Japanese. In Proceedings of the Tokyo conference on psycholinguistics, vol. 3, ed. Y. Otsu, 1-25. Tokyo: Hituzi Syobo.

Križ, M. 2015. Aspects of homogeneity in the semantics of natural language. $\mathrm{PhD}$ thesis, University of Vienna.

Križ, M., and E. Chemla. 2015. Two methods to find truth-value gaps and their application to the projection problem of homogeneity. Natural Language Semantics 23: 205-248. 
Križ, M., and B. Spector. 2017. Interpreting plural predication: Homogeneity and non-maximality. Unpublished manuscript, ENS.

Landman, F. 1998. Plurals and maximalization. In Events and grammar, ed. S. Rothstein, 237-272. Dordrecht: Kluwer Academic Publishers.

Magri, G. 2009. A theory of individual-level predicates based on blind mandatory scalar implicatures. $\mathrm{PhD}$ thesis, MIT.

Marty, P., and J. Romoli. 2019. Presupposed free choice and the theory of scalar implicatures. Unpublished manuscript, Leibniz-ZAS and Ulster University.

Meyer, M.-C. 2013. Ignorance and grammar. PhD thesis, MIT.

Meyer, M.-C. 2018. An apple or a pear: Free choice disjunction. In Wiley's semantics companion, ed. L. Matthewson, et al. New York: Wiley.

Romoli, J., and P. Santorio. 2019. Filtering free choice. Semantics and Pragmatics 12: 12.

Rothschild, D., and S. Yablo. 2018. Permissive updates. Unpublished manuscript, UCL and MIT.

Santorio, P. 2018. Alternatives and truthmakers in conditional semantics. Journal of Philosophy 115 (10): 513-549.

Santorio, P., and J. Romoli. 2017. Probability and implicatures: A unified account of the scalar effects of disjunction under modals. Semantics and Pragmatics 10 (13).

Simons, M. 2005. Semantics and pragmatics in the interpretation of 'or'. In Proceedings of SALT 15, ed. E. Georgala and J. Howell, 205-222. Ithaca, NY: CLC Publications, Cornell University.

Spector, B. 2007. Aspects of the pragmatics of plural morphology: On higher-order implicatures. In Presupposition and implicature in compositional semantics, ed. U. Sauerland and P. Stateva. London: Palgrave.

Spector, B. 2014. Global positive polarity items and obligatory exhaustivity. Semantics and Pragmatics 7: $1-61$.

Starr, W. 2016. Expressing permission. Proceedings of SALT 26: 325-349.

Tieu, L., C. Bill, and J. Romoli. 2018. Homogeneity or implicature: An experimental investigation of free choice. Unpublished manuscript, Western Sydney University, Leibniz-ZAS, and Ulster University.

Tieu, L., J. Romoli, P. Zhou, and S. Crain. 2016. Children's knowledge of free choice inferences and scalar implicatures. Journal of Semantics 33 (2): 269-298.

Willer, M. 2017. Widening free choice. Proceedings from the Amsterdam colloquium 2017. Available at http://events.illc.uva.nl/AC/AC2017/Proceedings/.

Zimmerman, T.E. 2000. Free choice disjunction and epistemic possibility. Natural Language Semantics 8: 255-290.

Publisher's Note Springer Nature remains neutral with regard to jurisdictional claims in published maps and institutional affiliations.

\section{Affiliations}

\section{Nicole Gotzner ${ }^{1} \cdot$ Jacopo Romoli $^{2}$ (D) Paolo Santorio ${ }^{3}$}

Jacopo Romoli

j.romoli@ulster.ac.uk

Paolo Santorio

paolosantorio@gmail.com

1 Leibniz-Zentrum Allgemeine Sprachwissenschaft, Shützenstr. 18, 10117 Berlin, Germany

2 University of Ulster, Jordanstown Campus Shore Road, BT370QB, United Kingdom

3 Department of Philosophy, University of Maryland, College Park 4300, Chapel Drive, College Park, MD 20742, USA 